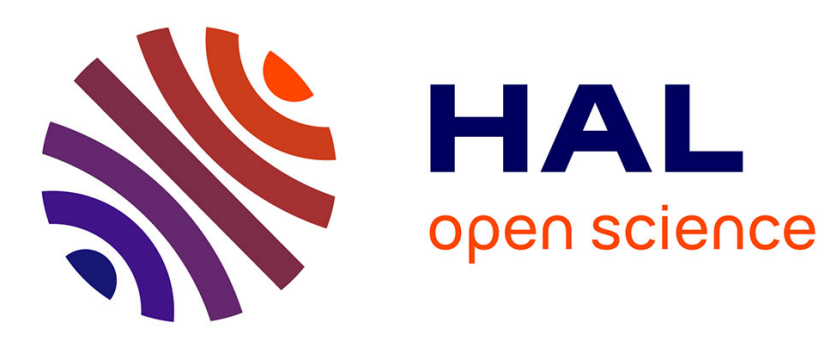

\title{
A mixed interface finite element for cohesive zone models
}

E. Lorentz

\section{To cite this version:}

E. Lorentz. A mixed interface finite element for cohesive zone models. Computer Methods in Applied Mechanics and Engineering, 2008, 198 (2), pp.302-317. 10.1016/j.cma.2008.08.006 . hal-03503253

\section{HAL Id: hal-03503253 \\ https: / hal-edf.archives-ouvertes.fr/hal-03503253}

Submitted on 27 Dec 2021

HAL is a multi-disciplinary open access archive for the deposit and dissemination of scientific research documents, whether they are published or not. The documents may come from teaching and research institutions in France or abroad, or from public or private research centers.
L'archive ouverte pluridisciplinaire HAL, est destinée au dépôt et à la diffusion de documents scientifiques de niveau recherche, publiés ou non, émanant des établissements d'enseignement et de recherche français ou étrangers, des laboratoires publics ou privés. 


\title{
A mixed interface finite element for cohesive zone models
}

\author{
E. Lorentz \\ Laboratoire de Mécanique des Structures Industrielles Durables, UMR CNRS/EDF 2832, 1 av. Général de Gaulle, 92141 Clamart cedex, France.
}

\begin{abstract}
The phenomena of crack initiation, propagation and ultimate fracture are studied here under the following assumptions: (i) the crack law is modelled by means of a cohesive zone model and (ii) the crack paths are postulated a priori. In this context, a variational formulation is proposed which relies on an augmented Lagrangian. A mixed interface finite element is introduced to discretise the crack paths, the degrees of freedom of which consist in the displacement on both crack lips and the density of cohesive forces. This enables an exact treatment of multi-valued cohesive laws (e.g. initial adhesion, contact conditions, possible rigid unloading, etc.), without penalty regularisation.

A special attention is paid to the convergence with mesh-refinement, i.e. the well-posedness of the problem, on the basis of theoretical results of contact mechanics and some complementary numerical investigations. Fulfilment of the LBB condition is the key factor to gain the desired properties. Moreover, it is shown that the integration of the constitutive law admits a unique solution as soon as some condition on the augmented Lagrangian is enforced. Finally, a 3D simulation shows the applicability to practical engineer problems, including in particular the robustness of the formulation and its compatibility with classical solution algorithms (Newton method, line-search, path-following techniques, ...).

Keywords: fracture mechanics ; cohesive zone model ; interface finite element ; mixed finite element ; augmented Lagrangian
\end{abstract}

\section{INTRODUCTION}

Various approaches are available to predict the fracture of industrial components submitted to excessive loading, ranging from failure criteria to non local damage constitutive laws. In order to be introduced in engineering simulation practices, they should meet a compromise between computational robustness, efficiency and physical accuracy. Other characteristics may also facilitate the process: consistency with former practices, simplicity, limitation of algorithmic parameters, applicability to various configurations, etc. In this context, the use of cohesive laws in combination with mixed interface finite elements is suggested, on the basis of the considerations exposed below.

\section{Cohesive zone models as an enhancement of the Griffith theory}

According to [1,2], the description of crack initiation and propagation should rely at least on two material parameters (for instance a critical stress and a fracture energy) in order to (i) cover the 
range of initially sound structures and cracked ones and (ii) correct the spurious scale effects observed with the Griffith theory [3]. Cohesive zone models, which go back to the pioneering work of Dugdale [4] and Barenblatt [5], constitute a sound and well-tried framework to respond this demand. Thus, they provide minimal improvements to overcome the limitations of the Griffith theory, they describe both stages of initiation and propagation, they take into account potential crack closure, they do not require the costly spatial refinement necessary with non local constitutive laws.

\section{The question of crack path prediction}

Cohesive zone models have been given a variational setting [6,7] in terms of minimisation of an energy, which implicitly encompasses crack path prediction. However, the corresponding discretisation and minimisation procedures need great care in order to ensure convergence with mesh refinement and remain complex [8,9]. To circumvent the difficulty, an alternative consists in following the propagation of individual cracks, a subject that has been paid much attention for the last fifteen years. Though attractive, the approach must fulfil complex and interconnected demands in order to be predictive: construction of a geometrically continuous crack path which goes across pre-existing finite elements $[10,11]$, derivation of a crack orientation criterion which provides a satisfactory direction as soon as damage occurs [12], applicability of the criterion in complex 3D situations with possibly coarse mesh [13], continuity of the structural response with respect to time [14], stability of the numerical scheme for crack propagation [15]. Even though specific numerical strategies have emerged that should cope with the full problem, see $[13,16]$ for instance, the fact that it is still a very active research field yet shows that fulfilment of all the previous demands remains a complex task. A straightforward application in engineering practices seems premature. Therefore, in what follows, the crack path is postulated a priori and not predicted by the computation. In spite of this major restriction, many industrial applications still enter the scope of the article in practice, especially for small propagations or interfacial cracks.

\section{Multi-valued cohesive laws and penalty methods}

Cohesive laws for cracking usually incorporate a stress threshold, contact conditions (crack closure) and possibly rigid unloading branches. On a mathematical ground, this results in multivalued functions relating the displacement gap to cohesive stresses, or equivalently to non 
continuous differentiable energies. Note that this feature is not restricted to initial adhesion (prior to local cohesive zone inception) but may also appear any time after the cohesive regime is triggered (unloading, closure): circumventing the difficulty by introducing discontinuous elements after the threshold is reached does not solve every case. A regularisation of the function is often proposed in the literature, based on a (quadratic) penalty method (an infinite stiffness is replaced by an extremely high one). Though attractive, special attention has to be paid to the quadrature rule in order to avoid spurious stress oscillations, see [11,17] for interface elements. Namely, the usual Gaussian quadrature rule lacks the necessary properties to ensure convergence with combined mesh refinement and penalty [18]. Moreover, a penalty stiffness may result in ill-conditioning of the global stiffness matrix, leading to increasing computational costs with penalty. At last, as soon as the energy is no more convex (which is the case of cohesive laws), the solution is not necessarily continuous with respect to the penalty parameter, raising the crucial question of how to choose the latter. Even though operational, penalty methods seem to need great care and expertise, and may not be adequate for common engineering practices. Therefore, we suggest not to regularise the problem but to eliminate the displacement discontinuity at a local level through a decomposition coordination method [19].

\section{Discretisation of displacement discontinuities}

Under the assumption of a priori knowledge of the potential crack paths, a straightforward method of discretisation consists in explicitly meshing the crack paths with interface finite elements. Application of the above-mentioned decomposition - coordination method then results in a mixed interface element which is the purpose of the article : its unknowns are the displacements (quadratic shape functions) and the cohesive force densities (linear shape functions). This extends the ideas of mortar elements [20], also widely applied to contact problems (a special case of cohesive law), see for instance [21].

Other discretisation techniques could have been considered, which would have avoided the explicit meshing of the crack path. Extended finite elements (X-FEM) [22,23] introduce global unknowns for the displacement discontinuity. However, avoiding penalty regularisation appears more difficult than for interface elements. Even though some promising results have been obtained for linear tetrahedrons in the treatment of contact conditions [24], the discretisation of the contact 
pressure remains heuristic and may fail to fulfil the LBB condition. Alternatively, embedded discontinuity finite elements (E-FEM) [25,26] rely on a local (i.e. at the element level) discretisation of the displacement discontinuity which can be eliminated from the global system by static condensation, hence avoiding penalty [27]. But the stability condition (patch-test) requires a non symmetrical formulation. Moreover, presentations of E-FEM in the literature generally rely on a linear interpolation of the displacement field and a constant jump inside each element, resulting in poor convergence rates with mesh refinement. Even though the extension to non constant jumps has been proposed in [28] to circumvent locking phenomena, higher degree interpolations would raise the question of the algorithmic implementation to perform the static condensation. Regarding the advantages and drawbacks of each family of elements, we choose to privilege the mixed interface finite element. We think that the other families are better suited for the more difficult problem of crack path prediction.

The article is organised as follows. Section 2 shows how the application of the decomposition coordination technique to cohesive problems leads in a natural way to the definition of a mixed finite element based on an augmented Lagrangian. Section 3 details the resulting integration of a special cohesive law which possesses all the basic features to be representative of more complex laws: perfect initial bonding, mixed-mode fracture, irreversibility, crack closure, stress-free ultimate fracture). Then, the implementation is validated by a test problem which admits a closed-form solution. Section 4 is dedicated to numerical analyses of the convergence with mesh refinement. Finally, a 3D application is shown that highlights the potency of the approach.

\section{CONSTRUCTION OF A MIXED INTERFACE FINITE ELEMENT}

\subsection{Preliminary: main assumptions}

In order to focus on the interface elements without additional complexity, the following assumptions are postulated from now on:

- postulated crack path. As stated in the introduction, the crack path is considered given a priori and not a result of the computation. Thus it is explicitly meshed with interface elements; 
- infinitesimal perturbation. In particular, the displacements should keep small enough so that geometrically coincident points on both sides of the crack path be considered remaining coincident. This is reasonable in the context of brittle fracture;

- elastic bulk behaviour. Definition of the interface element is independent of the constitutive law inside the bulk material. However, for the sake of simplicity, the numerical simulations are led with an elastic bulk behaviour. The only non linearity then results from the cohesive law.

\subsection{Modelling fracture as an energy minimisation}

In their approach of cohesive fracture mechanics, Francfort and Marigo [6] describe the state of a structure $\Omega$ through the displacement field $\mathbf{u}$ which may admit discontinuities $\boldsymbol{\delta}=\llbracket \mathbf{u} \rrbracket$ across surfaces $\Gamma(\mathbf{u})$. For a given load, the actual displacement is characterised by an optimality condition: it is a local minimum of the potential energy, expressed as the sum of the elastic strain energy, the cohesive energy and the work of external forces :

$$
\begin{gathered}
\mathrm{E}_{\mathrm{pot}}(\mathbf{u})=\mathrm{E}_{\mathrm{el}}(\mathbf{u})+\mathrm{E}_{\mathrm{fr}}(\llbracket \mathbf{u} \rrbracket)-\mathrm{W}_{\mathrm{ext}}(\mathbf{u}) \\
\mathrm{E}_{\mathrm{el}}(\mathbf{u})=\int_{\Omega / \Gamma(\mathbf{u})} \Phi(\boldsymbol{\varepsilon}(\mathbf{u})) d \Omega \quad ; \quad \mathrm{E}_{\mathrm{fr}}(\boldsymbol{\delta})=\int_{\Gamma(\mathbf{u})} \Pi(\boldsymbol{\delta}) d \Gamma
\end{gathered}
$$

where $\varepsilon$ is the strain tensor, $\Phi$ the (bulk) strain energy density and $\Pi$ the (surface) cohesive energy density. In such a presentation, the fracture mechanisms are considered reversible. In the next section, some irreversibility will be introduced through the dependence of the cohesive energy on internal variables. In that case, the loading path has to be discretised, but the solution over a given load increment is still characterised by (1), i.e. a minimisation with respect to the displacement field. It is remarkable that all features of the problem are deduced from the minimisation step, namely:

- cohesive law. It relates the displacement discontinuity $\boldsymbol{\delta}$ to the traction vector $\mathbf{t}$ through (generalised) differentiation of $\Pi$;

- contact conditions. They are enforced by means of the cohesive energy which involves an indicator function that precludes interpenetrations. 
- stress criterion for crack inception. Its derivation is detailed in [7]. Note that it is reduced to $\boldsymbol{\sigma}=\mathbf{0}$ as soon as the cohesive energy density $\Pi$ is differentiable at $\boldsymbol{\delta}=0$. Therefore, the non differentiability of $\Pi$ at $\boldsymbol{\delta}=0$ is an essential requirement to get a non zero stress threshold;

- crack path prediction. Indeed, all possible discontinuous displacements may be considered;

However, considering all possible discontinuous displacement lead to severe computational difficulties related to the discretisation of the functional space $B D(\Omega)$. In particular, allowing discontinuities across each finite element is not a converging process and thus it results in mesh dependency. Some attempts have been led, based either on a regularisation of discontinuities [8] or on mesh adaptation [9], but they are restricted to Griffith surface energy and still result in complex computations. Hence the assumption of postulated crack path: potential displacement discontinuities appear only across surfaces $\Gamma$ which are postulated a priori and do no more depend on $\mathbf{u}$.

\subsection{Derivation of a saddle-point problem through a decomposition - coordination method}

Even though the crack path is postulated, a straightforward minimisation of the potential energy is out of reach because of the non differentiability of the cohesive energy. In order to circumvent the difficulty, a decomposition - coordination technique [19] is introduced which confines the non differentiability to a local level (Gauss points)

\subsubsection{Augmented Lagrangian}

The relation between the discontinuity field $\boldsymbol{\delta}$ and the displacement field $\mathbf{u}$ is explicitly introduced into the minimisation process. Consider the energy $\mathrm{E}$ which depends explicitly on both $\mathbf{u}$ and $\boldsymbol{\delta}$ :

$$
\mathrm{E}(\mathbf{u}, \boldsymbol{\delta})=\int_{\Omega \backslash \Gamma} \Phi(\boldsymbol{\varepsilon}(\mathbf{u})) d \Omega-\mathrm{W}_{\mathrm{ext}}(\mathbf{u})+\int_{\Gamma} \Pi(\boldsymbol{\delta}) d \Gamma
$$

Minimisation of the potential energy (1) is then equivalent to the following constraint problem, where it is implicitly assumed that the displacement should belong to the set of kinematically admissible displacements:

$$
\min _{\substack{\mathbf{u}, \mathbf{\delta} \\ \llbracket \mathbf{u} \rrbracket \rrbracket \mathbf{\delta}}} \mathrm{E}(\mathbf{u}, \boldsymbol{\delta})
$$


The linear constraint $\llbracket \mathbf{u} \rrbracket=\boldsymbol{\delta}$ is treated by dualisation: a saddle point $(\mathbf{u}, \boldsymbol{\delta}, \boldsymbol{\lambda})$ of the following Lagrangian $\mathcal{L}$, where $\lambda$ denotes the field of Lagrange multipliers, corresponds to a solution $(\mathbf{u}, \boldsymbol{\delta})$ of (3):

$$
\mathcal{L}(\mathbf{u}, \boldsymbol{\delta}, \boldsymbol{\lambda}) \underset{\text { def. }}{=} \mathrm{E}(\mathbf{u}, \boldsymbol{\delta})+\int_{\Gamma} \lambda \cdot(\llbracket \mathbf{u} \rrbracket-\boldsymbol{\delta}) d \Gamma
$$

In order to gain some coercivity with respect to $\boldsymbol{\delta}$ which will prove necessary hereafter, a penalty term is finally added, without influence on the solutions since it is equal to zero for a fulfilled constraint. This results in the augmented Lagrangian $\mathcal{L}_{r}$, with $r$ the penalty coefficient:

$$
\mathcal{L}_{r}(\mathbf{u}, \boldsymbol{\delta}, \boldsymbol{\lambda}) \underset{\text { def. }}{=} \mathrm{E}(\mathbf{u}, \boldsymbol{\delta})+\int_{\Gamma} \boldsymbol{\lambda} \cdot(\llbracket \mathbf{u} \rrbracket-\boldsymbol{\delta}) d \Gamma+\int_{\Gamma} \frac{r}{2}(\llbracket \mathbf{u} \rrbracket-\boldsymbol{\delta})^{2} d \Gamma
$$

\section{Remark:}

In the context of adhesion $(\boldsymbol{\delta}=0, \Pi=0)$, an alternative is proposed in [29]. It relies on a discontinuous Galerkin method to enforce weakly the constraint $\llbracket \mathbf{u} \rrbracket=0$. It results in finding the stationary point of the following functional with respect to $\mathbf{u}$ :

$$
\mathrm{J}(\mathbf{u})=\mathrm{E}(\mathbf{u}, 0)+\int_{\Gamma} \boldsymbol{\Sigma}(\mathbf{u}) \cdot \llbracket \mathbf{u} \rrbracket d \Gamma+\int_{\Gamma} \frac{r}{2} \llbracket \mathbf{u} \rrbracket^{2} d \Gamma \quad \text { with } \quad \boldsymbol{\Sigma}(\mathbf{u})=\frac{\boldsymbol{\sigma}^{1}(\mathbf{u}) \cdot \mathbf{n}+\boldsymbol{\sigma}^{2}(\mathbf{u}) \cdot \mathbf{n}}{2}
$$

where $\boldsymbol{\sigma}^{1}$ and $\boldsymbol{\sigma}^{2}$ denote respectively the stress field on both sides of $\Gamma$ and $\mathbf{n}$ is its local normal. $\boldsymbol{\Sigma}(\mathbf{u})$ appears as an average stress vector. A comparison of the expressions (5) and (6) shows that the Lagrange multiplier is actually replaced by the average stress vector. Therefore, it avoids the introduction of the additional unknowns $\lambda$ but requires the computation of $\boldsymbol{\Sigma}(\mathbf{u})$ which is not common in the context of displacement-based finite elements and may depend on the local topology of the mesh. Extension to non linear cohesive laws seems to remain an open question.

\subsubsection{Example : application to contact problems}

It has been shown in [30] that in the case of pure contact conditions (no cohesive force), the minimisation with respect to $\boldsymbol{\delta}$ admits a closed-form solution which coincides with the augmented Lagrangian initially introduced in [31]. Indeed, the function $\Pi$ reduces to an indicator function in that case :

$$
\Pi(\boldsymbol{\delta})=\mathrm{I}_{\mathbb{R}^{+}}\left(\delta_{n}\right)=\left\{\begin{array}{cl}
0 & \text { if } \delta_{n} \geq 0 \\
+\infty & \text { if } \delta_{n}<0
\end{array}\right.
$$


where the subscript $n$ denotes the component normal to the contact surface $\Gamma\left(\delta_{n}=\boldsymbol{\delta} \cdot \mathbf{n}\right.$ is the opening displacement, $\mathbf{n}$ is the normal to $\Gamma$ ). The indicator function precludes interpenetration since negative displacement jumps would result in an infinite energy (hence not a minimum). A straightforward minimisation of the augmented Lagrangian with respect to $\boldsymbol{\delta}$ leads to the following expression (in which the MacCauley brackets $\langle x\rangle$ denote the positive part of a scalar $x$ ):

$$
\min _{\boldsymbol{\delta}} \mathcal{L}_{r}(\mathbf{u}, \boldsymbol{\delta}, \boldsymbol{\lambda})=\mathrm{E}_{\mathrm{el}}(\mathbf{u})-\mathrm{W}_{\mathrm{ext}}(\mathbf{u})+\frac{1}{2 r} \int_{\Gamma}\left[\left\langle-r \llbracket u_{n} \rrbracket-\lambda_{n}\right\rangle^{2}-\lambda_{n}^{2}\right] d \Gamma
$$

It is exactly the augmented Lagrangian introduced in [31]. This result suggests that some properties obtained for mixed duality-penalty contact methods may be extended to the present cohesive approach, in particular the choice of adequate finite elements.

\subsubsection{Characterisation of the saddle-point}

The solution algorithm will not deal with the inequality characterisation of a saddle-point but with the variational optimality conditions. For the augmented Lagrangian (5), the latter read:

$$
\begin{gathered}
\forall \delta \boldsymbol{\delta} \int_{\Gamma}[\mathbf{t}-\lambda-r(\llbracket \mathbf{u} \rrbracket-\boldsymbol{\delta})] \cdot \delta \boldsymbol{\delta} d \Gamma=0 \quad \text { with } \quad \mathbf{t} \in \partial \Pi(\boldsymbol{\delta}) \\
\forall \delta \mathbf{u} \int_{\Omega \backslash \Gamma} \boldsymbol{\sigma}: \boldsymbol{\varepsilon}(\boldsymbol{\delta} \mathbf{u}) d \Omega+\int_{\Gamma}[\lambda+r(\llbracket \mathbf{u} \rrbracket-\boldsymbol{\delta})] \cdot \llbracket \delta \mathbf{u} \rrbracket d \Gamma=\mathrm{W}_{\text {ext }}(\delta \mathbf{u}) \quad \text { with } \quad \boldsymbol{\sigma}=\frac{\partial \Phi}{\partial \boldsymbol{\varepsilon}}(\boldsymbol{\varepsilon}) \\
\forall \delta \boldsymbol{\lambda} \int_{\Gamma}(\llbracket \mathbf{u} \rrbracket-\boldsymbol{\delta}) \cdot \delta \lambda d \Gamma=0
\end{gathered}
$$

The equation (9) enforces the cohesive constitutive law. Signification of the subgradient $\partial \Pi$ will be given in section 3; at this stage, it is sufficient to say that $\mathbf{t} \in \partial \Pi(\boldsymbol{\delta})$ means that $\mathbf{t}$ and $\boldsymbol{\delta}$ are related by the cohesive constitutive law. Therefore, the equation (9) provides an interpretation for the Lagrange multiplier $\lambda$ : except for the penalty term, the Lagrange multiplier measures the cohesive forces. The equation (10) expresses the equilibrium inside the bulk and along the discontinuity surface $\Gamma$. Finally, the equation (11) enforces the constraint between the displacement field and its discontinuities. 


\subsection{Finite element discretisation}

As the crack path $\Gamma$ is postulated and thanks to the infinitesimal perturbation assumption, the spatial discretisation of the system (9)-(11) may rely on a simple kind of mortar segment-tosegment element. As depicted in figure 1, consider that the sub-domains $\Omega_{-}$and $\Omega_{+}$(the parts of $\Omega$ respectively below and above $\Gamma$ ) are discretised by tetrahedra or hexahedra so that the nodes on both sides of $\Gamma$ coincide. Note that this is not a stringent demand for a meshing algorithm, at least for simple shapes for $\Gamma$, namely planes. In that case, degenerated prisms or hexahedra can be used to discretise $\Gamma$ and relate both lips $\Gamma_{-}$and $\Gamma_{+}$of the potential cohesive crack.

Consider a given mesh, henceforth characterised by the subscript $h$ (maximal size of finite elements, for instance). A quadratic interpolation with classical Lagrange finite elements (P2continuous) is adopted inside the bulk. The space of discrete displacement fields $\mathcal{U}_{h}$ reads :

$$
\mathcal{U}_{h}=\{\mathbf{u} ; \forall \mathbf{x} \in \Omega \quad \mathbf{u}(\mathbf{x})=[\mathbf{N}(\mathbf{x})]\{\mathbf{U}\}\}
$$

where $\{\mathbf{U}\}$ denotes the displacement nodal vector and $[\mathbf{N}]$ the matrix of quadratic shape functions. The trace of the interpolated displacement on $\Gamma_{-}$and $\Gamma_{+}$is piecewise quadratic too, and such is the displacement discontinuity:

$$
\begin{gathered}
\forall \mathbf{s} \in \Gamma \quad \mathbf{u}_{\mid \Gamma_{-}}(\mathbf{s})=\left[\mathbf{N}_{-}(\mathbf{s})\right]\{\mathbf{U}\} \quad ; \quad \mathbf{u}_{\mid \Gamma_{+}}(\mathbf{s})=\left[\mathbf{N}_{+}(\mathbf{s})\right]\{\mathbf{U}\} \\
\forall \mathbf{s} \in \Gamma \quad \llbracket \mathbf{u}(\mathbf{s}) \rrbracket=[\mathbf{D}(\mathbf{s})]\{\mathbf{U}\} \quad \text { with } \quad[\mathbf{D}(\mathbf{s})]_{\text {def. }}^{=}\left[\mathbf{N}_{+}(\mathbf{s})\right]-\left[\mathbf{N}_{-}(\mathbf{s})\right]
\end{gathered}
$$

where $\left[\mathbf{N}_{-}\right]$and $\left[\mathbf{N}_{+}\right]$are the trace of $[\mathbf{N}]$ respectively on $\Gamma_{-}$and $\Gamma_{+}$and $[\mathbf{D}]$ the matrix for the quadratic shape functions which interpolates the displacement jump. Note that it is convenient to introduce a rotation into $[\mathbf{D}]$ to get the components of $\llbracket \mathbf{u} \rrbracket$ in a local co-ordinate system to distinguish the normal and the tangential parts.

The Lagrange multiplier field $\lambda$ is interpolated on $\Gamma$ by means of piecewise linear shape functions (P1-continuous), resulting in the space of discrete Lagrange multipliers $\mathfrak{L}_{h}$ :

$$
\mathfrak{L}_{h}=\{\lambda ; \forall \mathbf{s} \in \Gamma \quad \lambda(\mathbf{s})=[\mathbf{L}(\mathbf{s})]\{\mathbf{\Lambda}\}\}
$$

where $\{\boldsymbol{\Lambda}\}$ collects the nodal unknowns for the Lagrange multiplier and $[\mathbf{L}]$ is the matrix of linear shape functions on $\Gamma$. Thus, the constraint $\llbracket \mathbf{u} \rrbracket=\boldsymbol{\delta}$ enforced by (11) is only achieved in a weak sense. 
At last, the discretisation of the discontinuity field $\boldsymbol{\delta} \in \mathcal{D}_{h}$ is simply based on collocation points on $\Gamma$, of co-ordinates $\mathbf{s}_{g}$. They are chosen to be the integration points of the Gauss quadrature rule with three (triangles) or four (quadrangles) points by element. Actually, this corresponds to a P1discontinuous interpolation of $\boldsymbol{\delta}$. The weights of the Gauss points are denoted $\omega_{g}$; they will be used to compute the integrals in (9)-(11). The spatial discretisation, the corresponding notation and pictures of the finite elements are given in table 1.

The field $\boldsymbol{\delta}$ may vanish from the global formulation by means of static condensation. Indeed, with such a discretisation, (9) results in the exact satisfaction of the cohesive constitutive relation at each collocation point :

$$
\mathbf{t}_{g}=\boldsymbol{\lambda}_{g}+r\left(\llbracket \mathbf{u}_{g} \rrbracket-\boldsymbol{\delta}_{g}\right) \in \partial \Pi\left(\boldsymbol{\delta}_{g}\right) \quad \text { with }\left\{\begin{array}{rll}
\llbracket \mathbf{u}_{g} \rrbracket=\left[\mathbf{D}_{g}\right]\{\mathbf{U}\} & ;\left[\mathbf{D}_{g}\right]=\left[\mathbf{D}\left(\mathbf{s}_{g}\right)\right] \\
\boldsymbol{\lambda}_{g}=\left[\mathbf{L}_{g}\right]\{\boldsymbol{\Lambda}\} & ;\left[\mathbf{L}_{g}\right]=\left[\mathbf{L}\left(\mathbf{s}_{g}\right)\right]
\end{array}\right.
$$

Integration of the constitutive relation, i.e. solution of (16) as detailed in the next section, allows to compute $\boldsymbol{\delta}_{g}$ as a function (denoted $\widehat{\boldsymbol{\delta}}$ ) of $\{\mathbf{U}\}$ and $\{\boldsymbol{\Lambda}\}$ :

$$
\mathbf{t}_{g}=\boldsymbol{\lambda}_{g}+r\left(\llbracket \mathbf{u}_{g} \rrbracket-\boldsymbol{\delta}_{g}\right) \in \partial \Pi\left(\boldsymbol{\delta}_{g}\right) \quad \Leftrightarrow \quad \boldsymbol{\delta}_{g}=\hat{\boldsymbol{\delta}}\left(\llbracket \mathbf{u}_{g} \rrbracket, \lambda_{g}\right)=\widehat{\boldsymbol{\delta}}(\mathbf{U}, \boldsymbol{\Lambda})
$$

The penalty parameter $r$ will be adjusted so that the solution $\boldsymbol{\delta}_{g}$ to (16) be unique, whatever $\{\mathbf{U}\}$ and $\{\boldsymbol{\Lambda}\}$. This is a demand to ensure robustness.

The introduction of (17) into (10) and (11) then provides the non linear system the unknowns of which are the nodal displacements $\{\mathbf{U}\}$ and the nodal Lagrange multipliers $\{\boldsymbol{\Lambda}\}$ :

$$
\begin{gathered}
\int_{\Omega \backslash \Gamma}[\nabla \mathbf{N}]^{\mathrm{T}}: \hat{\boldsymbol{\sigma}}(\mathbf{U}) d \Omega+\sum_{g} \omega_{g}\left[\mathbf{D}_{g}\right]^{\mathrm{T}} \cdot\left(\left[\mathbf{L}_{g}\right]\{\boldsymbol{\Lambda}\}+r\left[\mathbf{D}_{g}\right]\{\mathbf{U}\}-r \widehat{\boldsymbol{\delta}}(\mathbf{U}, \boldsymbol{\Lambda})\right)=\left\{\mathrm{F}_{\text {ext }}\right\} \\
\sum_{g} \omega_{g}\left[\mathbf{L}_{g}\right]^{\mathrm{T}} \cdot\left(\left[\mathbf{D}_{g}\right]\{\mathbf{U}\}-\widehat{\boldsymbol{\delta}}(\mathbf{U}, \boldsymbol{\Lambda})\right)=0
\end{gathered}
$$

The bulk integral and the nodal vector of external forces are computed in a classical way. Finally, this system is solved simultaneously with respect to $\{\mathbf{U}\}$ and $\{\boldsymbol{\Lambda}\}$ by means of a (generalised) Newton method, where the tangent operator is symmetrical since (18)-(19) correspond to a saddlepoint problem.

Other solution techniques could have been considered to find the saddle-point of the augmented Lagrangian (5), depending in which order the variables $\mathbf{u}, \boldsymbol{\delta}$ and $\boldsymbol{\lambda}$ are treated. Some alternatives are proposed in [19] based on Uzawa type algorithms. In particular, one of them corresponds to the 
LATIN method proposed in [32] and is based on the following loop scheme: (i) minimisation with respect to $\boldsymbol{\delta}$ (the local non linear problem), (ii) updating the Lagrange multiplier $\lambda$ by a gradient method, (iii) minimisation with respect to $\mathbf{u}$ (the global linear problem) and (iv) updating again $\lambda$. Complementary highlights about this proposition can be found in [33]. In [30], another alternative is suggested : (i) combined minimisation with respect to $(\mathbf{u}, \boldsymbol{\delta})$, then (ii) actualisation of $\boldsymbol{\lambda}$ by a gradient method. The latter may also be replaced by a BFGS method, see [34] in another context. Among the many solution techniques, our choice of the Newton method as in [31] is motivated by:

- its good scalability with respect to increasing number of degrees of freedom,

- its classical algorithmic setting in pre-existing finite element software,

- its compatibility with other algorithmic procedures (line-search, path-following methods, etc.).

\subsection{Some insights towards mathematical investigations}

A precise mathematical analysis of the mixed formulation of the cohesive problem is out of the scope of the article. In this part, we only aim at gaining some confidence regarding the choice of the augmented Lagrangian and the spatial discretisation. Most of the comments hereafter are based on analyses dedicated to contact problems. Therefore, they are only hints for the comprehension of cohesive problems.

\subsubsection{Appropriate functional space for the Lagrange multiplier}

Thanks to the bulk elasticity, (each component of) the displacement field belongs to the space $H^{1}\left(\Omega_{-}\right) \times H^{1}\left(\Omega_{+}\right)$. Hence, the displacement discontinuity lies in the space $H^{1 / 2}(\Gamma)$. And to fulfil the LBB condition for the continuous problem, the space for the Lagrange multiplier is its dual, $H^{-1 / 2}(\Gamma)$, see [35]. Other choices such as $L^{2}(\Gamma)$ would not satisfy the LBB condition. However, according to [36], solution algorithms based on the augmented Lagrangian require at least the $L^{2}(\Gamma)$ regularity in order to avoid a dependency on the penalty parameter. Else, there is weak convergence towards the solution in $H^{-1 / 2}(\Gamma)$ with increasing penalty parameters. Fortunately, the effective solution mostly belongs to $L^{2}(\Gamma)$, except for very singular loading [36,18].

Therefore, regarding these possible limitations, a numerical analysis of the dependence on the penalty parameter and of the convergence is led in section 4 . 


\subsubsection{Choice of finite element interpolations}

The mixed finite element discretisation must satisfy stability conditions among which the discrete inf-sup one (LBB condition). This prescribes that the space for the discrete Lagrange multiplier should not be too large compared to the space for the discrete displacement along the crack [37]. In particular, a P1-discontinuous interpolation of $\boldsymbol{\lambda}$ with $\mathrm{P} 2$-continuous $\mathbf{u}$, which corresponds to the limit of penalty methods with a Gaussian quadrature rule, does not satisfy the inf-sup condition [35].

Actually, with a P2-continuous interpolation for $\mathbf{u}$, the following choices have been analysed in [35] which satisfy the inf-sup condition : P2/P2, P2/P1-continuous and P2/P0. Practical considerations then lead us to the choice P2/P1. Indeed, P2/P2 interpolations may produce oscillatory results for coarse meshes, even though the oscillations vanish with mesh refinement [18]. In addition, we observe that the numerical algorithm requires more iterations to converge than with a P2/P1 discretisation, a fact also reported in [18]. And that without benefit compared to the accuracy and convergence rate obtained with a P2/P1 element. On the other hand, P0 Lagrange multipliers hinder application of the Newton method because the tangent matrix is no more invertible. Moreover, they induce a loss of accuracy. Therefore, they are disregarded, which leaves us with the P2/P1-continuous finite element, probably the best choice for the considered problem.

\subsubsection{Stabilizing effect of penalty}

To ensure stability of the finite element formulation, another condition should be fulfilled : the ellipticity condition. On the contrary of the LBB condition, this one prescribes that the space for the discrete Lagrange multiplier should not be too small compared to the space for the discrete displacement along the crack [37]. Else, displacement oscillation modes may appear with mesh refinement. The condition is more complex to state in the case of non linear problems; in particular, the lack of convexity and coercivity of the cohesive energy adds some specificities.

We propose here a rough observation which highlights the role of the penalty coefficient. Consider the patch-test in which the displacement field $\mathbf{u}$ is linear with constant discontinuity:

$$
\llbracket \mathbf{u} \rrbracket=\boldsymbol{\delta}^{0} \text { constant }
$$


This displacement field is introduced in the discrete problem (17)-(19); a solution $\boldsymbol{\delta}=\boldsymbol{\delta}^{0}$ is expected to pass the patch-test. Unfortunately, depending on the penalty parameter, this is not necessarily true. Indeed, the weak constraint (19) only enforces:

$$
\boldsymbol{\delta} \in \mathcal{D}_{h} \text { s.t. } \forall \delta \boldsymbol{\lambda} \in \mathfrak{L}_{h} \int_{\Gamma}\left(\boldsymbol{\delta}^{0}-\boldsymbol{\delta}\right) \cdot \delta \boldsymbol{\lambda} d \Gamma=0 \quad \Rightarrow \quad \boldsymbol{\delta}-\boldsymbol{\delta}^{0} \in \mathfrak{L}_{h}^{\perp}
$$

The vector space $\mathfrak{L}_{h}{ }^{\perp}$ is not reduced to $\{0\}$ because $\operatorname{dim} \mathfrak{L}_{h}<\operatorname{dim} \mathcal{D}_{h}$ (respectively P1-continuous and P1-discontinuous polynomials on $\Gamma$ ). Actually, $\mathfrak{L}_{h}{ }^{\perp}$ enables oscillations of $\boldsymbol{\delta}$ around $\boldsymbol{\delta}^{0}$. They should be controlled by the cohesive energy $\Pi$ since, in the present case, the discrete system (17)(19) may be reinterpreted as the following minimisation problem, with $\boldsymbol{\delta}=\boldsymbol{\delta}^{0}+\boldsymbol{\delta}^{1}$ :

$$
\text { Find } \boldsymbol{\delta}^{1} \in \mathfrak{L}_{h}{ }^{\perp} \text { minimising } \int_{\Gamma} \Pi_{r}\left(\boldsymbol{\delta}^{1}\right) d \Gamma \quad \text { with } \quad \Pi_{r}\left(\boldsymbol{\delta}^{1}\right)=\Pi\left(\boldsymbol{\delta}^{0}+\boldsymbol{\delta}^{1}\right)+\frac{r}{2} \boldsymbol{\delta}^{1} \cdot \boldsymbol{\delta}^{1}
$$

However, $\Pi$ is neither convex, nor coercive in $\mathfrak{L}_{h}{ }^{\perp}$ (section 3). Alone, it is not sufficient to control the oscillations in order to ensure $\boldsymbol{\delta}^{1}=0$. The role is devoted to the penalty term. Suppose that there exists a constant $H^{c}$ such that $\Pi_{r}$ is strictly convex (and coercive) for any $r>H^{c}$ (in the case of frictionless contact, one has $\left.H^{c}=0\right)$. Then, $\boldsymbol{\delta}^{1}=0$ is the only solution, thanks to convexity: the penalty term controls the oscillations. In addition, it will be checked in section 4 that for increasing mesh refinement, the solution does not depend on the value of $r>H^{c}$.

\section{INTEGRATION OF THE COHESIVE CONSTITUTIVE LAW}

The cohesive constitutive behaviour is totally defined by the cohesive energy density $\Pi(\boldsymbol{\delta})$. Even though the variational formulation of the previous section encompasses many choices of cohesive energy, we now focus on a specific cohesive model so as to illustrate the integration procedure and in particular how the non-differentiability should be treated. For the sake of simplicity, its only features are :

(i) contact conditions;

(ii) perfect initial bonding;

(iii) fracture triggered by both tension and shear;

(iv) total ultimate fracture, i.e. zero cohesive forces beyond some damage level;

(v) irreversibility of fracture. 
In particular, it means that there is no ultimate friction, nor distinction between tension and shear fracture mechanisms. Nevertheless, these features seem sufficient to evaluate the capabilities of the mixed finite element in realistic configurations, as shown in the next section where some simulations based on this model will be performed.

\section{Preliminary notations.}

Because of the non interpenetration constraint, the direction $\mathbf{n}$ normal to the crack path $\Gamma$ (opening/compression) has to be distinguished from the in-plane directions (sliding/shear). Therefore, the following notations are introduced, where $\mathbf{v}$ denotes any vector quantity:

$$
\mathbf{n} \cdot \mathbf{n}=1 \quad ; \quad v_{n}=\mathbf{v} \cdot \mathbf{n} \quad ; \quad \mathbf{v}_{/ /}=\mathbf{v}-v_{n} \mathbf{n} \quad ; \quad\langle\mathbf{v}\rangle_{+}=\left\langle v_{n}\right\rangle \mathbf{n}+\mathbf{v}_{/ /} ;\|\mathbf{v}\|_{+}=\left(\langle\mathbf{v}\rangle_{+} \cdot\langle\mathbf{v}\rangle_{+}\right)^{1 / 2}
$$

\subsection{The Talon - Curnier constitutive law}

The cohesive constitutive law proposed by Talon and Curnier [38] fulfils the five abovementioned demands and can easily be cast into the energetic framework (1). The responses of the model in pure tension and pure shear and the stress initiation criterion are plotted in figure 2. The corresponding cohesive energy is defined as follows.

First, tension and shear displacement discontinuities are combined in a unique scalar $\delta_{e q}$ which measures the magnitude of the discontinuity, hence fulfilling (iii). Here, a quadratic form is assumed for sake of simplicity:

$$
\delta_{e q}=\sqrt{\boldsymbol{\delta} \cdot \boldsymbol{\delta}}
$$

Then, irreversibility (v) is taken into account by means of an additional scalar internal variable $\kappa$ which measures the current maximal loading level:

$$
\kappa(t)=\sup _{t^{\prime}<t} \delta_{e q}\left(t^{\prime}\right)
$$

The cohesive energy depends on the internal variable $\kappa$ and on the equivalent displacement jump $\delta_{e q}$. Contact conditions (i) are taken care by an indicator function which restricts the normal discontinuity to $\delta_{n} \geq 0$ (opening):

$$
\Pi(\boldsymbol{\delta}, \kappa)=\mathrm{I}_{\mathbb{R}^{+}}\left(\delta_{n}\right)+\psi\left(\max \left(\delta_{e q}, \kappa\right)\right) \quad \text { with } \quad \psi: \mathbb{R}^{+} \rightarrow \mathbb{R}
$$


The function $\psi$ characterises namely the response to a monotonous pure mode I solicitation. Following [7], $\psi$ should be a differentiable increasing function, where $\sigma^{c}=\psi^{\prime}(0)$ defines the critical stress which parameterises the initiation criterion (ii). Moreover, stability of the fracture process requires that $\psi$ be concave [39]. And at last, the ultimate fracture demand (iv) is fulfilled as soon as $\psi$ reaches its upper bound $G^{c}$ for a finite value $\delta_{e q}=\delta^{c}$, where $G^{c}$ is the fracture energy and $\delta^{c}$ the critical opening beyond which cohesive forces vanish. Therefore, the following function $\psi$ is chosen, which corresponds to the characteristics depicted in figure 2 :

$$
\psi\left(\delta_{e q}\right)=\left\{\begin{array}{cc}
G^{c} \frac{\delta_{e q}}{\delta^{c}}\left(2-\frac{\delta_{e q}}{\delta^{c}}\right) & \text { if } \delta_{e q} \leq \delta^{c} \\
G^{c} & \text { if } \delta_{e q} \geq \delta^{c}
\end{array}\right.
$$

along with the relation between material parameters:

$$
G^{c}=\frac{1}{2} \sigma^{c} \delta^{c}
$$

At this stage, the cohesive energy and hence the constitutive law are totally defined. Nevertheless, it may be interesting to explicitly express the relation between the displacement discontinuity $\boldsymbol{\delta}$ and the tension vector $\mathbf{t}$, which is condensed in the following differential inclusion, where $\partial \Pi$ denotes the sub-gradient of $\Pi$ in the sense of Clarke [40]:

$$
\mathbf{t} \in \partial \Pi(\boldsymbol{\delta})
$$

For a given value of $\kappa$, a rough interpretation of the subgradient $\partial \Pi(\boldsymbol{\delta})$ is the set of slopes less steep than any directional derivative of $\Pi$ at $\boldsymbol{\delta}$ (all admissible directions are considered). Mathematically, it reads:

$$
\partial \Pi(\boldsymbol{\delta})=\left\{\mathbf{t} \in \mathbb{R}^{3} ; \forall \boldsymbol{v} \in \mathbb{R}^{3} \quad \mathbf{t} \cdot \mathbf{v} \leq \Pi^{\circ}(\boldsymbol{\delta}, \boldsymbol{v})\right\}
$$

where $\Pi^{\circ}(\boldsymbol{\delta}, \boldsymbol{v})$ is the directional derivative of $\Pi$ at $\boldsymbol{\delta}$ along the direction $\boldsymbol{v}$ :

$$
\Pi^{\circ}(\boldsymbol{\delta}, \boldsymbol{v})=\limsup _{\substack{\zeta \rightarrow 0^{+} \\ \mathbf{d} \rightarrow \boldsymbol{\delta}}} \frac{\Pi(\mathbf{d}+\zeta \mathbf{v})-\Pi(\mathbf{d})}{\zeta}
$$

This definition coincides with the gradient of $\Pi$ anywhere $\Pi$ is differentiable. Therefore, considering (26), points which deserve a special attention are $\boldsymbol{\delta}=0, \delta_{n}=0$ and $\delta_{e q}=\kappa$. Following the definition (30), the subgradient is determined by means of straightforward (but cumbersome) 
computations. Its expression depends on the considered point $(\boldsymbol{\delta}, \kappa)$, with four different domains corresponding to four different regimes of constitutive behaviour. It reads:

- point $(\boldsymbol{\delta}=\mathbf{0}, \kappa=0)$ : perfect adhesion, i.e. initiation criterion

$$
\partial \Pi(\boldsymbol{\delta})=\left\{\mathbf{t} \in \mathbb{R}^{3} ;\|\mathbf{t}\|_{+} \leq \sigma^{c}\right\}
$$

- domain where $\delta_{e q}<\kappa:$ crumpling

$$
\partial \Pi(\boldsymbol{\delta})=\left\{t_{n} \mathbf{n} ; t_{n} \leq 0 \text { and } \delta_{n} \geq 0 \text { and } t_{n} \delta_{n}=0\right\}
$$

- hyper-cone $\delta_{e q}=\kappa>0$ : unloading

$$
\partial \Pi(\boldsymbol{\delta})=\left\{t_{n} \mathbf{n}+\rho \boldsymbol{\delta} ; 0 \leq \rho \leq \frac{\psi^{\prime}(\kappa)}{\kappa} \text { and } t_{n} \leq 0 \text { and } \delta_{n} \geq 0 \text { and } t_{n} \delta_{n}=0\right\}
$$

- domain where $\delta_{e q}>\kappa:$ damage

$$
\partial \Pi(\boldsymbol{\delta})=\left\{t_{n} \mathbf{n}+\psi^{\prime}\left(\delta_{e q}\right) \frac{\boldsymbol{\delta}}{\delta_{e q}} ; t_{n} \leq 0 \text { and } \delta_{n} \geq 0 \text { and } t_{n} \delta_{n}=0\right\}
$$

\section{Remarks:}

1. There exists a domain for the cohesive force which corresponds to a zero discontinuity (32): it is indeed an initiation criterion, depicted in figure 2c. This is related to the non differentiability of $\Pi$ at $\boldsymbol{\delta}=0$. The shape of the domain depends directly on the expression of $\delta_{e q}$. Note that the domain is larger than the one exhibited in [7] because there is only one crack direction which is considered (normal to $\mathbf{n}$ ) instead of any possible direction.

2. The Kuhn and Tucker condition which appears in (33)-(35) means that in the case of possible contact $\left(\delta_{n}=0\right)$, the cohesive force is compressive $\left(t_{n} \leq 0\right)$ and the value of the compression is left undefined by the constitutive law.

3. There exists a gap between crumpling and damage conditions, that is the cohesive force is not continuous with respect to the displacement discontinuity (34).

4. In case of pure mode I or pure shear mode, the responses in figure 2 are retrieved. Note that the peak values in tension and in shear modes are equal because of the choice of the norm (24).

\subsection{Numerical integration}

According to (17), numerical integration of the constitutive relation consists in computing $\boldsymbol{\delta}_{g}$ for given values of $\llbracket \mathbf{u}_{g} \rrbracket$ and $\lambda_{g}$, and that for each Gauss point (from now on, the subscript $g$ is 
omitted). Thus, application of the mixed interface finite element leads to a constitutive law integration where forces are inputs (along with the displacement discontinuity $\llbracket \mathbf{u} \rrbracket$ and the internal variable $\kappa$ ) and displacement gaps are outputs, i.e. the reverse format of usual (primal) interface elements. Actually, (17) is a characterisation of the following minimum:

$$
\min _{\boldsymbol{\delta} \in \mathbb{R}^{3}}\left[\boldsymbol{\lambda} \cdot(\llbracket \mathbf{u} \rrbracket-\boldsymbol{\delta})+\frac{r}{2}(\llbracket \mathbf{u} \rrbracket-\boldsymbol{\delta})^{2}+\Pi(\boldsymbol{\delta}, \kappa)\right] \quad \Leftrightarrow \quad \lambda+r \llbracket \mathbf{u} \rrbracket-r \boldsymbol{\delta} \quad \in \quad \partial \Pi(\boldsymbol{\delta}, \kappa)
$$

Moreover, the evolution of the internal variable $\kappa$ should be involved. A discretisation of time is necessary. Consider a sequence of instants $t^{0}<t^{1}<\ldots<t^{n}$ and the corresponding quantities $\lambda^{n}$, $\llbracket \mathbf{u}^{n} \rrbracket, \boldsymbol{\delta}^{n}$ and $\kappa^{n}$. Application of an implicit scheme to discretise the model of irreversibility introduced in (25)-(26) results in a two-step iterative process [41]:

$$
\begin{gathered}
\boldsymbol{\delta}^{n}=\underset{\boldsymbol{\delta} \in \mathbb{R}^{3}}{\arg \min }\left[\lambda^{n} \cdot\left(\llbracket \mathbf{u}^{n} \rrbracket-\boldsymbol{\delta}\right)+\frac{r}{2}\left(\llbracket \mathbf{u}^{n} \rrbracket-\boldsymbol{\delta}\right)^{2}+\Pi\left(\boldsymbol{\delta}, \boldsymbol{\kappa}^{n-1}\right)\right] \\
\boldsymbol{\kappa}^{n}=\max \left(\kappa^{n-1}, \boldsymbol{\delta}_{e q}^{n}\right)
\end{gathered}
$$

Of course, the actualisation (38) does not raise any question; solving the discretised system (37)(38) then reduces to the problem (36) with $\kappa=\kappa^{n-1}$, hence a fixed parameter. A graphical interpretation of the differential inclusion in (36) is provided in figure 3 in the case of pure mode I without unloading : a solution is the intersection of the linear function $\boldsymbol{\delta} \mapsto \boldsymbol{\lambda}+r \llbracket \mathbf{u} \rrbracket-r \boldsymbol{\delta}$ with the graph $\partial \Pi\left(\delta, \kappa^{n-1}\right)$; in particular, the penalty coefficient $r$ is the (negative) slope of the linear function.

In order to achieve robustness of the integration, it is desirable that the function between brackets in (36) be strictly convex with respect to $\boldsymbol{\delta}$ so that the minimum be unique. This is equivalent to the strict convexity of the augmented function $\Pi_{r}$ introduced in (22). Therefore, it is not only a question of robustness, important by itself, but also of well-posedness to avoid oscillations as stated in subsection 2.5.3. Introducing $H^{c}$ the softening modulus of the constitutive relation, a sufficient condition for strict convexity is:

$$
r>\max _{x \geq 0}\left|\psi^{\prime \prime}(x)\right|=\frac{\sigma^{c}}{\delta^{c}}=H^{c}
$$

Its graphical interpretation is straightforward on figure 3 in the case of pure mode I without unloading. 


\section{Sketch of the proof.}

The indicator function is convex and does not need special attention. Regarding convexity, the penalty term can be reduced to a quadratic function of $\delta_{e q}$ (let us denote $\delta_{e q}=\mathrm{N}(\boldsymbol{\delta})$ for what follows). At last, the following property is applied, stating that a function go $\mathrm{N}$ is strictly convex if $\mathrm{N}$ is strictly convex (yes), g strictly increasing (yes with $r>0$ ) and $\mathrm{g}$ strictly convex (yes as soon as $r>H^{c}$, hence (39)).

From now on, it is assumed that (39) is satisfied. Then, the function between brackets in (36) is also coercive and semi-lower continuous, hence ensuring existence of a minimum. Finally, there exists a unique minimum if $r>H^{c}$.

Let us now focus on the numerical integration itself, that is the solution of (37) for a given value of $\boldsymbol{\tau}=\boldsymbol{\lambda}+r \llbracket \mathbf{u} \rrbracket$, i.e. the input quantity of the constitutive law. Thanks to the existence and uniqueness of the solution, it is interesting to take advantage of the characterisation (36) in terms of subgradient, the expressions of which are given in (32) to (35). Solving the problem in each case, four alternatives are retrieved:

- if $\kappa=0$ and $\|\boldsymbol{\tau}\|_{+} \leq \sigma^{c}=\psi^{\prime}(0)$ : perfect adhesion

$$
\boldsymbol{\delta}=0
$$

- if $\kappa>0$ and $\|\boldsymbol{\tau}\|_{+}<r \kappa:$ crumpling

$$
\boldsymbol{\delta}=\frac{\langle\boldsymbol{\tau}\rangle_{+}}{r}
$$

- if $\kappa>0$ and $r \kappa \leq\|\boldsymbol{\tau}\|_{+} \leq r \kappa+\psi^{\prime}(\kappa)$ : unloading

$$
\boldsymbol{\delta}=\kappa \frac{\langle\boldsymbol{\tau}\rangle_{+}}{\|\boldsymbol{\tau}\|_{+}}
$$

- if $r \kappa+\psi^{\prime}(\kappa)<\|\boldsymbol{\tau}\|_{+}:$damage

$$
\boldsymbol{\delta}=\delta_{e q} \frac{\langle\boldsymbol{\tau}\rangle_{+}}{\|\boldsymbol{\tau}\|_{+}} \quad ; \quad \delta_{e q} \text { solution of } \psi^{\prime}\left(\delta_{e q}\right)+r \delta_{e q}=\|\boldsymbol{\tau}\|_{+}
$$

Actually, the distinction whether $\kappa=0$ or not is unnecessary since (40) appears as a special case of

In conclusion, expressions of the solution $\boldsymbol{\delta}$ (output of the constitutive law) are provided in (40)(43) as functions of the input $\boldsymbol{\tau}=\boldsymbol{\lambda}+r \llbracket \mathbf{u} \rrbracket$. They correspond to the different regimes of the 
constitutive law. No numerical approximate solution is required, since the only equation to solve, in (43), is piecewise linear.

\subsection{Numerical validation by comparison to a closed-form solution}

To validate the implementation of both the mixed finite element and the cohesive constitutive law, a test problem is proposed which admits a closed-form solution. It consists of an elastic beam clamped at one end, submitted to a prescribed tension displacement (no bending) and glued on a rigid plane surface, see figure $4 \mathrm{a}$. The glue is modelled by the Talon - Curnier law reduced to mode II. The description of the problem is one dimensional. Three areas are observed which move with increasing prescribed displacement and correspond to perfect sticking (no debonding), a cohesive zone and a free surface (zero cohesive force).

As the loading displacement is monotonous, no local unloading is expected. Therefore, the displacement of the membrane is a minimum of the following energy:

$$
\begin{gathered}
\min _{u \in K A(U)}\left[\frac{E S}{2} \int_{0}^{L}\left(\frac{d u}{d x}\right)^{2} d x+\int_{0}^{L} \psi(|u|) w d x\right] \\
K A(U)=\left\{u \in H^{1}(] 0 L[) ; u(0)=0 \text { and } u(L)=U\right\}
\end{gathered}
$$

where $E$ is the elastic stiffness, $S$ the cross section area, $L$ the beam length, $w$ its width, $u$ the (horizontal) displacement field, $U$ the prescribed displacement at the end of the beam and $\psi$ the cohesive energy (27) relying on the characteristic length $\delta^{c}$ and the fracture energy $G^{c}$. To focus on significant features, the variables are normalised:

$$
\begin{aligned}
\bar{u}=\frac{u}{\delta^{c}} \quad ; \quad \bar{U}=\frac{U}{\delta^{c}} \quad ; \quad \bar{x} & =\frac{x}{D} \quad ; \quad \bar{L}=\frac{L}{D} \quad ; \quad \bar{\Pi}(\bar{u})=\frac{\psi\left(\left|\delta^{c} \bar{u}\right|\right)}{2 G^{c}}=\frac{\bar{u}}{2}(2-\bar{u}) \\
\text { with } \quad D & =\delta^{c} \sqrt{\frac{E S}{2 w G^{c}}}
\end{aligned}
$$

The minimisation problem (44) is then equivalent to:

$$
\begin{gathered}
\min _{\bar{u} \in K A(\bar{U})}\left[\frac{1}{2} \int_{0}^{\bar{L}}\left(\frac{d \bar{u}}{d \bar{x}}\right)^{2} d \bar{x}+\int_{0}^{\bar{L}} \bar{\Pi}(\bar{u}) d \bar{x}\right] \\
K A(\bar{U})=\left\{\bar{u} \in H^{1}(] 0 \bar{L}[) ; \bar{u}(0)=0 \text { and } \bar{u}(\bar{L})=\bar{U}\right\}
\end{gathered}
$$

The first order optimality conditions are necessary conditions for $\bar{u}$ to be a minimum: 


$$
\bar{\tau} \underset{\text { def. }}{=} \frac{d^{2} \bar{u}}{d \bar{x}^{2}} \in \partial \bar{\Pi}(\bar{u}) \quad ; \quad \bar{u} \in C^{1}(] 0 \bar{L}[) \quad ; \quad \bar{u}(0)=0 \quad ; \quad \bar{u}(\bar{L})=\bar{U}
$$

where $\bar{\tau}$ is the (normalised) cohesive force density, defined through the equilibrium equation. Note that the continuity of the derivative of $\bar{u}$ is obtained thanks to the bounded character of the subgradient $\partial \bar{\Pi}$, hence precluding any punctual cohesive force. On the basis of (32)-(35), the subgradient is actually equal to:

$$
\partial \bar{\Pi}(\bar{u})= \begin{cases}{[-1} & 1] \\ \{(1-|\bar{u}|) \operatorname{sgn} \bar{u}\} & \text { if } \bar{u}=0 \\ \{0\} & \text { if }|\bar{u}| \geq 1\end{cases}
$$

One can recognize the graph of figure $2 \mathrm{~b}$. The solution is then composed of several branches on contiguous intervals with $C^{1}$ continuity. Each branch belongs to one of the possible regimes:

- Perfect bonding

$$
\bar{u}=0
$$

- Progressive damage, i.e. cohesive zone

$$
\begin{array}{ll}
\bar{u}(\bar{x})=A \cos \bar{x}+B \sin \bar{x}+1 & \text { for } \bar{u}>0 \\
\bar{u}(\bar{x})=A \cos \bar{x}+B \sin \bar{x}-1 & \text { for } \bar{u}<0
\end{array} \quad A \in \mathbb{R}, B \in \mathbb{R},|\bar{u}(\bar{x})| \leq 1
$$

- Complete decohesion

$$
\bar{u}(\bar{x})=A \bar{x}+B \quad A \in \mathbb{R}, B \in \mathbb{R},|\bar{u}(\bar{x})| \geq 1
$$

In order to simplify the analysis, we assume from now on that the beam is sufficiently long, namely $\bar{L}>\bar{U}-1+\pi / 2$, and that the loading is high enough, i.e. $\bar{U}>1$. In that case, it is easy to show that there exists three areas, which correspond respectively to (49), (50) and (51), from left to right. Thanks to the $C^{1}$ continuity and the boundary conditions, the constants can be determined, leading to the following expression, with $\bar{b}$ the length of the beam yet left unaffected by decohesion:

$$
\bar{b}=\bar{L}-(\bar{U}-1)-\frac{\pi}{2} ;\left\{\begin{array}{lll}
0 \leq \bar{x} \leq \bar{b} & \bar{u}(\bar{x})=0 & \bar{\tau}(\bar{x})=0 \\
\bar{b} \leq \bar{x} \leq \bar{b}+\frac{\pi}{2} & \bar{u}(\bar{x})=1-\cos (\bar{x}-\bar{b}) & \bar{\tau}(\bar{x})=\cos (\bar{x}-\bar{b}) \\
\bar{b}+\frac{\pi}{2} \leq \bar{x} \leq \bar{L} & \bar{u}(\bar{x})=\bar{x}-\bar{L}+\bar{U} & \bar{\tau}(\bar{x})=0
\end{array}\right.
$$


As can be observed, the cohesive force density $\bar{\tau}$ belongs to the space $L^{2}$ but it is not continuous at $\bar{x}=\bar{b}$.

The couple $(\bar{u}, \bar{\tau})$ is one possible solution of the problem (47). In order to enhance the potency of the test case, a sufficient condition for the strict convexity of the energy in (46) is exhibited, so that $(\bar{u}, \bar{\tau})$ is actually its only minimum. First, the normalised energy in (46) is rewritten as:

$$
\frac{1}{2} \int_{0}^{\bar{L}}\left(\frac{d \bar{u}}{d \bar{x}}\right)^{2} d \bar{x}+\int_{0}^{\bar{L}} \bar{\Pi}(\bar{u}) d \bar{x}=\underbrace{\int_{0}^{\bar{L}}\left[\frac{1}{2}\left(\frac{d \bar{u}}{d \bar{x}}\right)^{2}-\frac{\beta}{2} \bar{u}^{2}\right] d \bar{x}}_{\mathrm{J}_{\mathrm{el}}(\bar{u})}+\underbrace{\int_{0}^{\bar{L}}\left[\bar{\Pi}(\bar{u})+\frac{\beta}{2} \bar{u}^{2}\right] d \bar{x}}_{\mathrm{J}_{\mathrm{fr}}(\bar{u})}
$$

where $\beta>0$ is a parameter left undetermined. The idea consists in taking advantage of the convexity of the strain energy to compensate the lack of convexity of the fracture energy. Thus, the first term $J_{e l}$ is a quadratic form. Its convexity is related to its positiveness; computation of the Rayleigh quotient shows that $\mathrm{J}_{\mathrm{el}}$ is convex as soon as $\beta \leq \pi^{2} / \bar{L}^{2}$ (first eigenvalue). The convexity of the second term $J_{\text {fr }}$ is established by considering its integrand: thanks to the expression (45) for $\bar{\Pi}, J_{\text {fr }}$ is strictly convex as soon as $\beta>1$. Consequently, the energy is strictly convex and its minimum unique if $1<\pi^{2} / \bar{L}^{2}$, that is $\bar{L}<\pi$.

A computation of the beam is performed with $\bar{U}=1.5$ and $\pi>\bar{L}>\bar{U}-1+\pi / 2$, so that $(\bar{u}, \bar{\tau})$ defined in (52) is the unique solution to (46). The mesh size is set such that 25 finite elements discretise the cohesive zone (of normalised length $\pi / 2$ ), while the penalty coefficient is taken equal to $100 \times \bar{H}^{c}=100$. The results plotted in figure 4 are in good agreement with the solution (52), even though oscillations of the Gibbs type are observed around the discontinuity of the cohesive force. Although the area over which they spread goes to zero in length with mesh refinement, they do not vanish in amplitude ( $L^{2}$ but not pointwise convergence). In conclusion, the implementation is validated. Questions of convergence are the purpose of the next section.

\section{NUMERICAL APPLICATIONS}

The section aims at demonstrating the capacities of the mixed interface finite element. Two structures are studied. The first one is bidimensional in order to enable an analysis of convergence rates with mesh refinement. The second one is qualitative and illustrates the contributions of the 
interface elements in 3D. The numerical simulations have been performed with the open source finite element software Code_Aster [42].

\subsection{Measure of convergence rates}

\subsubsection{Test problem}

Several questions have been raised in the mathematical study: convergence of the displacement field and of the cohesive forces with respect to mesh refinement, sensitivity to the penalty term and its influence on the solution algorithm. Some numerical investigations are led in this sense. They are based on the numerical study of sandwich beams the interface of which obeys the Talon Curnier law, see figure 5a for the geometry and the material parameters. In order to avoid that the convergence rates be polluted by possible singularities, a very smooth loading is applied, so that the optimal theoretical convergence rate $O\left(h^{2}\right)$ in elasticity (with $h$ the size of the quadratic triangles) is almost reached: indeed, the observed numerical convergence rate for the stress field is $O\left(h^{1.92}\right)$ in elasticity (i.e. without any interface). More precisely, the loading consists in the following body force $\mathbf{b}$ :

$$
\begin{gathered}
\mathbf{b}(x, y)=\gamma\left[2 b^{(1)}(x, y) \mathbf{e}_{\mathbf{x}}+0.08 b^{(0)}(x, y) \mathbf{e}_{\mathbf{y}}\right] \quad\left(\text { in N } / \mathrm{mm}^{3}\right) \\
0 \leq \gamma \leq 1 ;-L \leq x \leq L \quad ; \quad-h \leq y \leq h
\end{gathered}
$$

where $\gamma$ denotes the load magnitude, $b^{(0)}$ the unit vertical part corresponding to a "three point bending" and $b^{(1)}$ the unit horizontal part corresponding to torque at both ends, as pictured in red in figure 5a. Their expressions are given below and represented in figure $5 \mathrm{~b}$ for the right part of the beams (symmetry):

$$
\begin{array}{ll}
b^{(0)}(x, y)=\left(1-\tanh \frac{|x|}{h}\right) \sin \frac{\pi\langle-y\rangle}{h}- & \left(1-\tanh \frac{L-|x|}{h}\right) \sin \frac{\pi\langle y\rangle}{h} \\
b^{(1)}(x, y)= & \left(1-\tanh \frac{L-|x|}{h}\right) \sin \frac{2 \pi\langle y\rangle}{h}
\end{array}
$$

Thanks to the symmetry, only one half of the structure (right) is computed. The global response of the structure is plotted in figure $6 \mathrm{~b}$ in terms of the vertical displacement of point $\mathrm{P}$ (top right in figure 5a) versus the loading magnitude $\gamma$ ranging from 0 to 1 . A regime of initiation, followed by an instability (crack jump) and then a progressive propagation of the interfacial crack can be observed. The stress field (longitudinal and shear) for $\gamma=1$ is plotted on the deformed geometry in 
figure 6a, where the crack is apparent. Finally, the cohesive forces are plotted in figure 6c. Three zones can be distinguished, from left to right: adhesion (undamaged), cohesive zone and fracture. On the contrary of the test-case in section 3.3, it appears that the cohesive forces are continuous, so that there is no more oscillations.

\subsubsection{Convergence with respect to the mesh size}

In order to estimate the convergence rates, a sequence of meshes is built, each one being embedded in the former, with element size twice smaller. Five levels of meshes are considered to measure convergence rates, see figure $5 \mathrm{c}$ for an illustration of the two first levels (in red and black). As no closed-form solution is available for the problem, the convergence rate is estimated by comparing two successive solutions. Consider for instance the sequence of solution stress fields $\boldsymbol{\sigma}_{n}$, with $n$ the level of mesh refinement ( $h_{n}=h_{0} 2^{-n}$ is the corresponding size of the finite elements). Then the following estimate is used:

$$
\left\|\sigma_{n}-\sigma_{\infty}\right\|=O\left(h_{n}^{s}\right) \Rightarrow\left\|\sigma_{n+1}-\sigma_{n}\right\|=O\left(h_{n}^{s}\right)
$$

The gaps between two successive solutions are plotted in figure $7 \mathrm{a}$ and figure $7 \mathrm{~b}$, respectively $\left\|\boldsymbol{\sigma}_{n+1}-\boldsymbol{\sigma}_{n}\right\|_{L^{2}(\Omega)}$ for the stress field and $\left\|\lambda_{n+1}-\lambda_{n}\right\|_{L^{2}(\Gamma)}$ for the cohesive force field. In both cases, the convergence is observed. The convergence rate $s$ is equal to 1.9 for the stress field: the mixed interface finite elements do not introduce any degradation of the convergence compared to the elastic problem. And the convergence rate for the cohesive force is equal to 1.15. These results are in reasonable agreement with the theoretical propositions in [18], thanks to the fulfilment of the LBB condition.

\subsubsection{Influence of penalty}

In order to show that the solution does not depend on the penalty parameter $r$, at least asymptotically, three values of $r$ have been considered: $r \in \mathcal{R}=\left\{10 H^{c}, 100 H^{c}, 1000 H^{c}\right\}$, where $H^{c}$ is the softening modulus introduced in (39). We recall that the penalty term reads:

$$
P_{r}=\int_{\Gamma} \frac{r}{2}(\llbracket \mathbf{u} \rrbracket-\delta)^{2} d \Gamma
$$

The convergence of the penalty term with the mesh size is showed in figure 7c: it vanishes with mesh refinement. It proves that the space for the Lagrange multiplier is rich enough to ensure alone 
fulfilment of the constraint $\llbracket \mathbf{u} \rrbracket=\boldsymbol{\delta}$. Indeed, the maximal gap between solutions obtained with different penalty parameters in the sampling set $\mathcal{R}$ also goes to zero with mesh refinement, see figure $7 \mathrm{~d}$ :

$$
\begin{array}{ll}
\max _{\substack{r \in \mathcal{R} \\
r^{\prime} \in \mathcal{R}}}\left\|\boldsymbol{\sigma}_{n}(r)-\boldsymbol{\sigma}_{n}\left(r^{\prime}\right)\right\|_{L^{2}(\Omega)} \stackrel{n \rightarrow \infty}{\longrightarrow} 0 \\
\max _{\substack{r \in \mathcal{R} \\
r^{\prime} \in \mathcal{R}}}\left\|\lambda_{n}(r)-\lambda_{n}\left(r^{\prime}\right)\right\|_{L^{2}(\Gamma)} \stackrel{ }{\longrightarrow} \quad \stackrel{n \rightarrow \infty}{\longrightarrow} 0
\end{array}
$$

It means that the sensitivity with respect to the penalty parameter vanishes with mesh refinement.

However, the penalty parameter may have an influence on the convergence of Newton algorithm, as shown in figure 8. Actually, the use of a line-search technique has been necessary to enforce the convergence of the algorithm with $r=1000 H^{c}$. On the contrary, we have observed that the line-search slowed down the convergence for $r=10 \mathrm{H}^{c}$, in particular for the finest mesh. Finally, it appears that using the intermediate value $r=100 H^{c}$ results in a rather stable convergence whatever the mesh size and is not perturbed by the line-search (which could reveal necessary to overcome possible instabilities). It is difficult to generalise the proposition. Nevertheless, it seems that $r=100 H^{c}$ introduces a sufficient level of coercivity in order to avoid that Newton algorithm escapes its attractor, without resulting in ill-conditioned problems. Additional simulations would be necessary to confirm this rule of thumb.

\subsection{Application to a 3D structure}

Finally, the study of a 3D structure is performed to demonstrate the full applicability of the mixed interface finite element. As previously, the structure is made of glued sandwich beams, as described in figure 9, where the interface is described with the Talon - Curnier law. The loading consists in bending prescribed displacements in two directions. More precisely, the left end of the upper beam is free, its right end is clamped except for the longitudinal displacement which must remain a constant field along the cross section, the value of which is free:

$$
\text { upper beam : } \frac{\partial u}{\partial y}(L, y, z)=\frac{\partial u}{\partial z}(L, y, z)=0 \quad ; \quad v(L, y, z)=0 \quad ; \quad w(L, y, z)=0
$$

The special condition on $u$ sets the rotation to zero but enables a longitudinal displacement in order to limit $\sigma_{x z}$ shear. The right end of the lower beam is free, while its left end is totally submitted to prescribed displacements: 
lower beam : $u(0, y, z)=0 ; v(0, y, z)=\gamma \times 1 \mathrm{~mm} \quad ; \quad w(0, y, z)=\gamma \times 1 \mathrm{~mm}$

where $\gamma$ denotes again the normalised load magnitude. Actually, the vertical bending is expected stable because of contact conditions (a sudden fracture of the interface does not release the amount of elastic energy since the beams remain partially bent). On the contrary, the horizontal bending is expected unstable because the beams are free to retrieve their straight shape in this direction in case of total fracture of the interface. Therefore, the chosen loading is thought to trigger the following features: active contact conditions along a moving surface, coupled shear - opening fracture modes, full 3D mechanisms resulting in non straight cohesive zones inside the interface plane and a final global instability of the structure.

The spatial discretisation of the beams is based on quadratic regular hexahedra of edge length $6.25 \mathrm{~mm}$. The interface is discretised by means of the corresponding mixed interface elements. It results in about 300000 degrees of freedom, which makes the structure realistic and representative of real industrial problems. The penalty parameter is set to $100 H^{c}$ on the basis of the experience acquired in section 4.1. Regarding the loading conditions, a monotonous control of the magnitude $\gamma$ is not possible because of the expected instability. To avoid a dynamic simulation, a path following method is applied, as proposed in [43]. $\gamma$ is controlled so that during each increment, the damage (i.e. the normalised cohesive energy $\psi / G^{c}$ ) progress at most of a given quantity set equal to 0.1 in the simulation. It means that for each increment, there exists a point of the interface where the damage increases of 0.1; elsewhere, the damage increase is less than 0.1 . The computation is led up to complete fracture of the interface. 75 load increments are required, each of them with 4 Newton iterations in average: the convergence of the solution algorithm is satisfactory, both in stable and unstable regimes.

The global response of the structure figure 10 shows the expected phases: progressive damage of the interface followed by an unstable propagation up to complete failure, characterised by a sharp snap back of the force - displacement curve. It should be noted that no small spurious snap-backs appear, on the contrary of what is observed with usual interface elements $[43,44]$. Actually, embedded discontinuity finite elements do not either exhibit such spurious snap-backs [41]. Therefore, it seems reasonable to relate them to the penalty regularisation of initial adhesion, another reason to enforce perfect initial bonding. Two load levels are selected for the oncoming 
post-treatments: one during the progressive propagation $(\gamma \approx 1$, stage A on the curve), the other corresponding to the peak load ( $\gamma \approx 2.9$, stage $\mathbf{B}$ on the curve).

The deformed shape and the longitudinal stress $\sigma_{x x}$ representative of bending are pictured in figure 11a. They illustrate the distribution of stress responsible for the decohesion: the free ends of the beams tend to retrieve their straight shapes (minimal elastic energy) and thus apply shear and opening forces on the interface. Moreover, the contact of the lower beam on the upper beam enforces a global (vertical) bending, whatever the state of the interface. The damage field along the interface $\psi / G^{c}$ is plotted in figure 11b. Thanks to the exact enforcement of initial adhesion and ultimate decohesion, a clear identification of the cohesive zone is possible as the points where $0<\psi / G^{c}<1$. It appears that the crack front adopts a complex shape which evolves during propagation, due to $3 \mathrm{D}$ effects. At last, figure 11c pictures the proportion between opening and shear modes inside the cohesive zone on the basis of the following local indicator:

$$
m=\frac{\left\|\mathbf{t}_{/ /}\right\|^{2}}{\|\mathbf{t}\|_{+}^{2}}
$$

The indicator takes the value 0 for pure opening mode and 1 for pure shear mode (with possible compression). Of course, a value in-between denotes a combination of both modes. It appears that the full range of solicitations takes place in the simulation, even though shear mode is dominant, especially when the peak load is reached.

Finally, this 3D simulation seems to prove that mixed interface finite elements are sufficiently robust to address industrial applications and provide several kinds of pertinent information about the fracture process.

\section{SUMMARY}

A mixed interface finite element has been proposed to model cohesive zones along given paths, in full compatibility with usual 3D finite elements for the bulk behaviour. Its unknowns are nodal displacements on both lips of the cohesive crack and nodal Lagrange multipliers, interpreted as the surface density of cohesive forces. The Lagrangian of the problem is then augmented in order to gain a convexity property. In that way, integration of the cohesive law is reduced to the computation 
of local displacement discontinuities corresponding to local cohesive forces, a problem that admits a unique solution, whatever the cohesive forces.

In conclusion, this approach suffers from the following limitations and drawbacks:

- Because the method relies on interface elements, the potential crack paths have to be postulated a priori.

- Additional degrees of freedom are introduced, corresponding to the cohesive forces. However, their number remains low since they are restricted to the potential crack paths, which are surfaces (resp. lines) in 3D (resp. 2D).

- The introduction of Lagrange multipliers leads to a mixed problem: the solution is characterised as a saddle-point and no more a minimum as in the initial energetic formulation. Consequently, some tools of mathematical optimisation are no more available to solve the problem.

- The Lagrangian has to be augmented in order to gain a local convexity property. This implies the introduction of a penalty parameter, without sensitivity for the continuous problem but which may affect the results of the spatially discrete problem. Nevertheless, this dependence vanishes with mesh refinement. Moreover, the numerical examples show that this sensitivity remains small.

- The local integration of the constitutive equations relies on the expression of the cohesive law in the reverse format [cohesive forces $\rightarrow$ displacement discontinuity], which is unusual when considering interface elements.

Conversely, the limitations and drawbacks are counterbalanced by the following attractive properties:

- No regularisation (penalty) of the cohesive law is required, regarding namely initial adhesion, contact conditions and possibly rigid unloading. In particular, this avoids ill-conditioning. It is illustrated by an application to the Talon-Curnier cohesive law which exhibits regimes of perfect adhesion, rigid unloading and crumpling.

- The choice of a quadratic discretisation for the displacements and a linear discretisation for the Lagrange multipliers fulfils the LBB condition. In turn, it ensures convergence of the solution with mesh refinement in terms of displacements and cohesive forces, as checked numerically. 
- The convergence rates that would be obtained without any interface is not perturbed by the presence of mixed interface elements.

- Thanks to the saddle-point characterisation, the consistent tangent matrix is symmetric.

- The proposed element is fully compatible with common solution algorithms such as the Newton method, line-search accelerations, path-following techniques, etc. This has been proved through 2D and 3D computations which demonstrate the applicability, the robustness and the efficiency of the mixed interface element.

\section{REFERENCES}

1. Leguillon D. (2002) Strength or toughness ? A criterion for crack onset at a notch. Eur. J. Mech. A/Solids 21, 6172.

2. Marigo J.-J., Truskinovsky L. (2004) Initiation and propagation of fracture in the models of Griffith and Barenblatt. Continuum Mech. Thermodyn. 16, 391-409.

3. Wadier Y., Lorentz E. (2004) Mécanique de la rupture fragile en présence de plasticité : modélisation de la fissure par une entaille. C. R. Mécanique 332, 979-986.

4. Dugdale D.S. (1960) Yielding of steel sheets containing slits. J. Mech. Phys. Solids 8, 100-104.

5. Barenblatt G.I. (1962) The mathematical theory of equilibrium cracks in brittle fracture. Adv. Appl. Mech. 7, 55129.

6. Francfort G., Marigo J.-J. (1998) Revisiting brittle fracture as an energy minimization problem. J. Mech. Phys. Solids 46(8), 1319-1342.

7. Charlotte M., Laverne J., Marigo J.-J. (2006) Initiation of cracks with cohesive force models: a variational approach. European Journal of Mechanics A/Solids 25, 649-669.

8. Bourdin B., Francfort G.A., Marigo J.-J. (2000) Numerical experiments in revisitted brittle fracture. J. Mech. Phys. Solids 48(4), 797-826.

9. Fraternali F. (2007) Free discontinuity finite element models in two-dimensions for in-plane crack problems. Theoretical and Applied Fracture Mechanics 47, 274-282.

10. Alfaiate J., Wells G.N., Sluys L.J. (2002) On the use of embedded discontinuity element with crack path continuity for mode I and mixed-mode fracture. Engineering Fracture Mechanics 69, 661-686.

11. De Borst R. (2003) Numerical aspects of cohesive-zone models. Engineering Fracture Mechanics 70, 1743-1757.

12. Jirasek M., Zimmermann T. (2001) Embedded crack model : Part II: Combination with smeared cracks. Int. J. Numer. Meth. Engng. 50, 1291-1305.

13. Gasser T., Holzapfel G. (2006) 3D crack propagation in unreinforced concrete. A two-step algorithm for tracking 3D crack paths. Comput. Methods Appl. Mech. Engrg. 195, 5198-5219.

14. Sam C.-H., Papoulia K.D., Vavasis S.A. (2005) Obtaining initially rigid cohesive finite element models that are temporally convergent. Eng. Fract. Mech. 72, 2247-2267.

15. Réthoré J., Gravouil A., Combescure A. (2005) An energy-conserving scheme for dynamic crack growth using the eXtended finite element method. Int. J. Num. Meth. Engng. 63(5), 631-659.

16. Oliver J., Huespe A.E. (2004) Theoretical and computational issues in modelling material failure in strong discontinuity scenarios. Comput. Methods Appl. Mech. Engrg. 193, 2987-3014.

17. Schellekens J.C.J., de Borst R. (1993) On the numerical integration of interface elements, 36, 43-66.

18. Fortin M. Error estimates and contact problems. Courtesy of Pr. Fortin, GIREF internal report, université Laval, Québec.

19. Fortin M., Glowinski R. Augmented lagrangian methods : application to the numerical solution of boundary-value problems. Studies in mathematics and its applications 15, North-Holland, 1983.

20. Ben Belgacem F. (1999) The mortar finite element method with Lagrange multipliers. Numer. Math. 84, 173-197.

21. Puso M.A., Laursen T.A. (2004) A mortar segment-to-segment frictional contact method for large deformations. Comp. Meth. Appl. Mech. Engng. 193, 4891-4913. 
22. Moes N., Dolbow J., Belytschko T. (1999) A finite element method for crack growth without remeshing. Int. J. Num. Meth. Engng. 46, 131-150.

23. Hansbo A., Hansbo P. (2004) A finite element method for the simulation of strong and weak discontinuities in solid mechanics. Comput. Methods Appl. Mech. Engrg. 193, 3523-3540.

24. Geniaut S., Massin P., Moes N. (2007) A stable 3D contact formulation for cracks using X-FEM. Eur. J. Comp. Mech. 16(1).

25. Jirasek M. (2000) Comparative study on finite elements with embedded discontinuities. Comput. Methods Appl. Mech. Engng. 188, 307-330.

26. Oliver J., Huespe A.E., Sanchez P.J. (2006) A comparative study on finite elements for capturing strong discontinuities: E-FEM vs. X-FEM. Comput. Methods Appl. Mech. Engrg. 195, 4732-4752.

27. Jirasek M., Zimmermann T. (2001) Embedded crack model : I. Basic formulation. Int. J. Numer. Meth. Engng. 50, 1269-1290.

28. Manzoli O.L., Shing P.B. (2006) A general technique to embed non-uniform discontinuities into standard solid finite elements. Comp. Struct. 84, 742-757.

29. Mergheim J., Kuhl E., Steinmann P. (2004) A hybrid discontinuous Galerkin / interface method for the computational modelling of failure. Comm. Num. Meth. Engng. 20, 511-519.

30. Fortin M., Tardieu N., Chamberland E. (2005) Un algorithme de sous-gradient pour le traitement du contact frottant. Proceedings «7è colloque national en calcul des structures », Giens, France.

31. Alart P., Curnier A. (1991) A mixed formulation for frictional contact problems prone to Newton like solution methods. Comp. Meth. Appl. Mech. Engng. 92, 353-375.

32. Ladeveze P. Mécanique non linéaire des structures. Hermes, 1996.

33. Doyen D. (2008) Modélisation et simulation de la propagation quasi-statique des fissures - livrable 1. EDF R\&D internal report, SINETICS/2008, cow. Duwig V., Ern A., Piperno S.

34. Lorentz E., Benallal A. (2005) Gradient constitutive relations: numerical aspects and application to gradient damage. Comput. Methods Appl. Mech. Engrg. 194, 5191-5220.

35. Bathe K.J., Brezzi F. (2001) Stability of finite element mixed interpolation for contact problems. Rend. Mat. Acc. Lincei 12(9), 167-183.

36. Stadler G. (2007) Path-following and augmented Lagrangian method for contact problems in linear elasticity. Journal of computational and applied mathematics 203, 533-547.

37. Bathe K.J. (2001) The inf-sup condition and its evaluation for mixed finite element methods. Computer and structures 79, 243-252.

38. Talon C., Curnier A. (2003) A model of adhesion coupled to contact and friction. Eur. J. Mech. A/Solids 22(4), 545-565.

39. Del Piero G., Truskinovsky L. (2001) Macro- and micro- cracking in one dimensional elasticity. Int. J. Solids Struct. 38, 1135-1148.

40. Clarke F.H. Optimization and non smooth analysis. Classics in applied mathematics 5, SIAM, 1990.

41. Laverne J. (2004) Formulation énergétique de la rupture par des modèles de forces cohésives : considérations théoriques et implantations numériques. Thèse de doctorat de l'université Paris XIII.

42. Code_Aster. Open source finite element software available on www.code-aster.org, courtesy of EDF.

43. Lorentz E., Badel P. (2004) A new path-following constraint for strain-softening finite elements simulations. Int. J. Num. Meth. Engng. 60, 499-526.

44. Alfano G., Crisfield M.A. (2001) Finite element interface models for the delamination analysis of laminated composites : mechanical and computational issues. Int. J. Num. Meth. Engng. 50(7), 1701-1736. 


\section{LIST OF FIGURES AND TABLES}

figure 1 - Discretisation by interface finite elements

figure 2 - Talon - Curnier cohesive law

figure 3 - Solution of the constitutive equation

figure 4 - Validation test: an elastic beam glued on a rigid surface

figure 5 - Description of the 2D sandwich beams problem

figure 6 - Decohesion of the 2D sandwich beams

figure 7 - Sensitivity to mesh size and penalty parameter

figure 8 - Convergence of Newton algorithm with respect to mesh size and penalty parameter

figure 9 - Description of the 3D sandwich beams problem

figure 10 - Global response of the 3D sandwich beams

figure 11 - Decohesion of the 3D sandwich beams : local analyses

table 1 - Spatial discretisation 


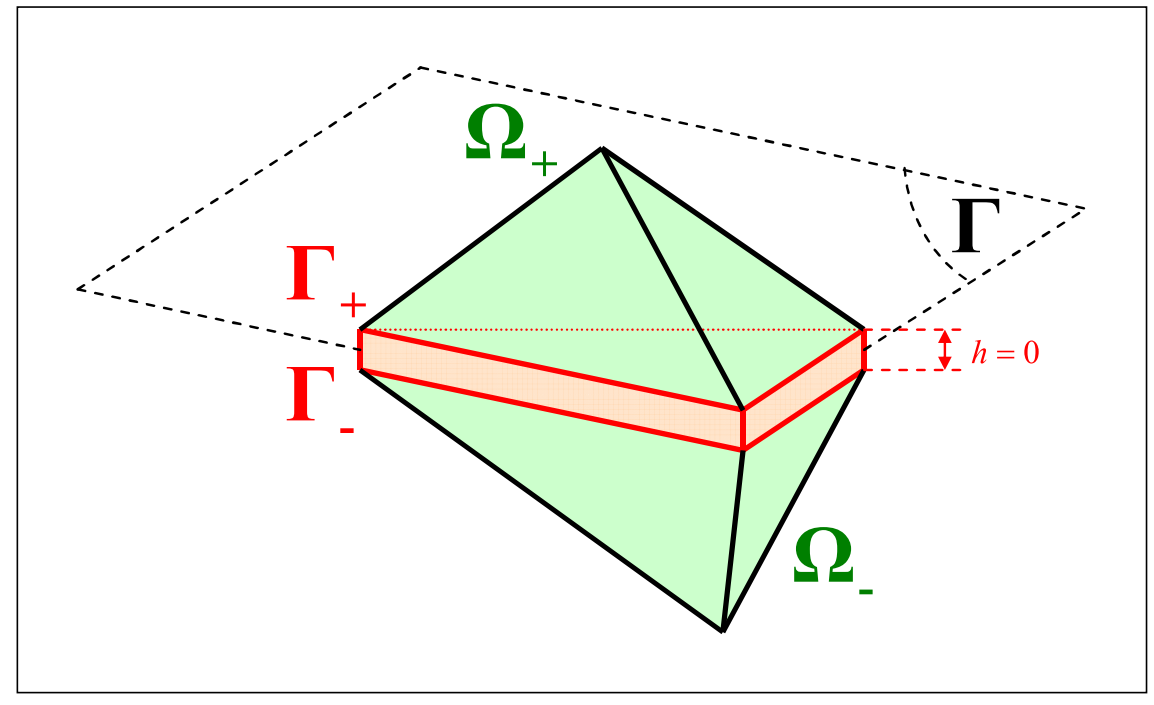

figure 1 - Discretisation by interface finite elements 


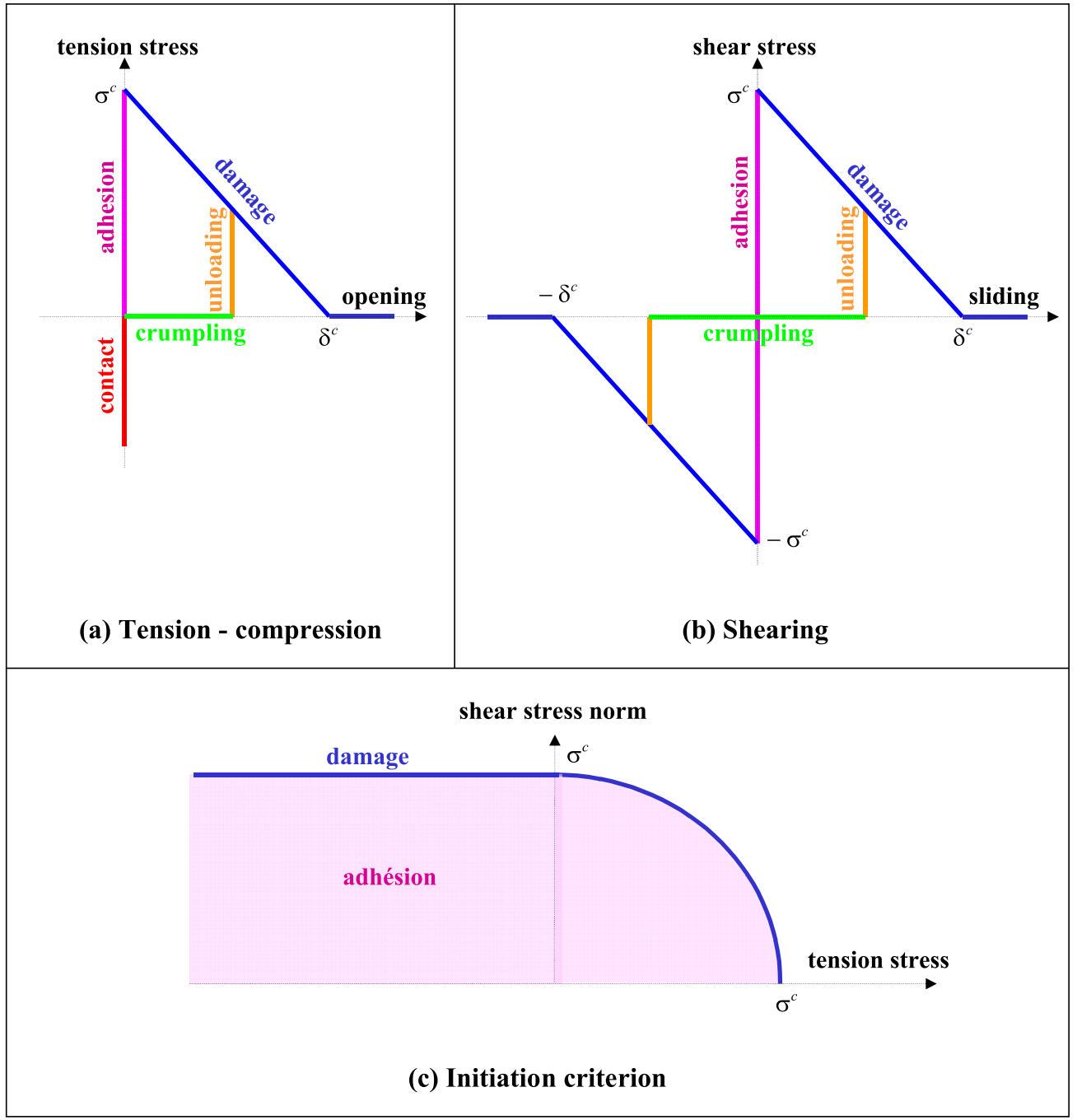

figure 2-Talon - Curnier cohesive law 
Figure 3 - Solution of the constitutive equation

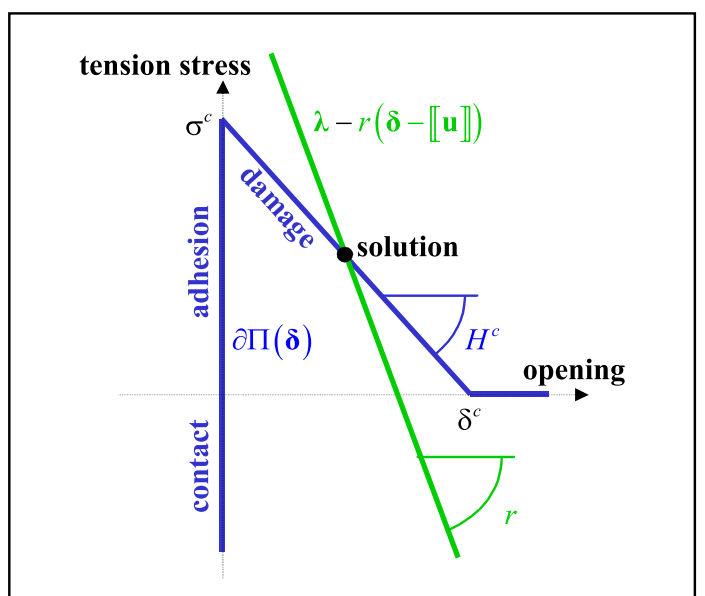

figure 3 - Solution of the constitutive equation 


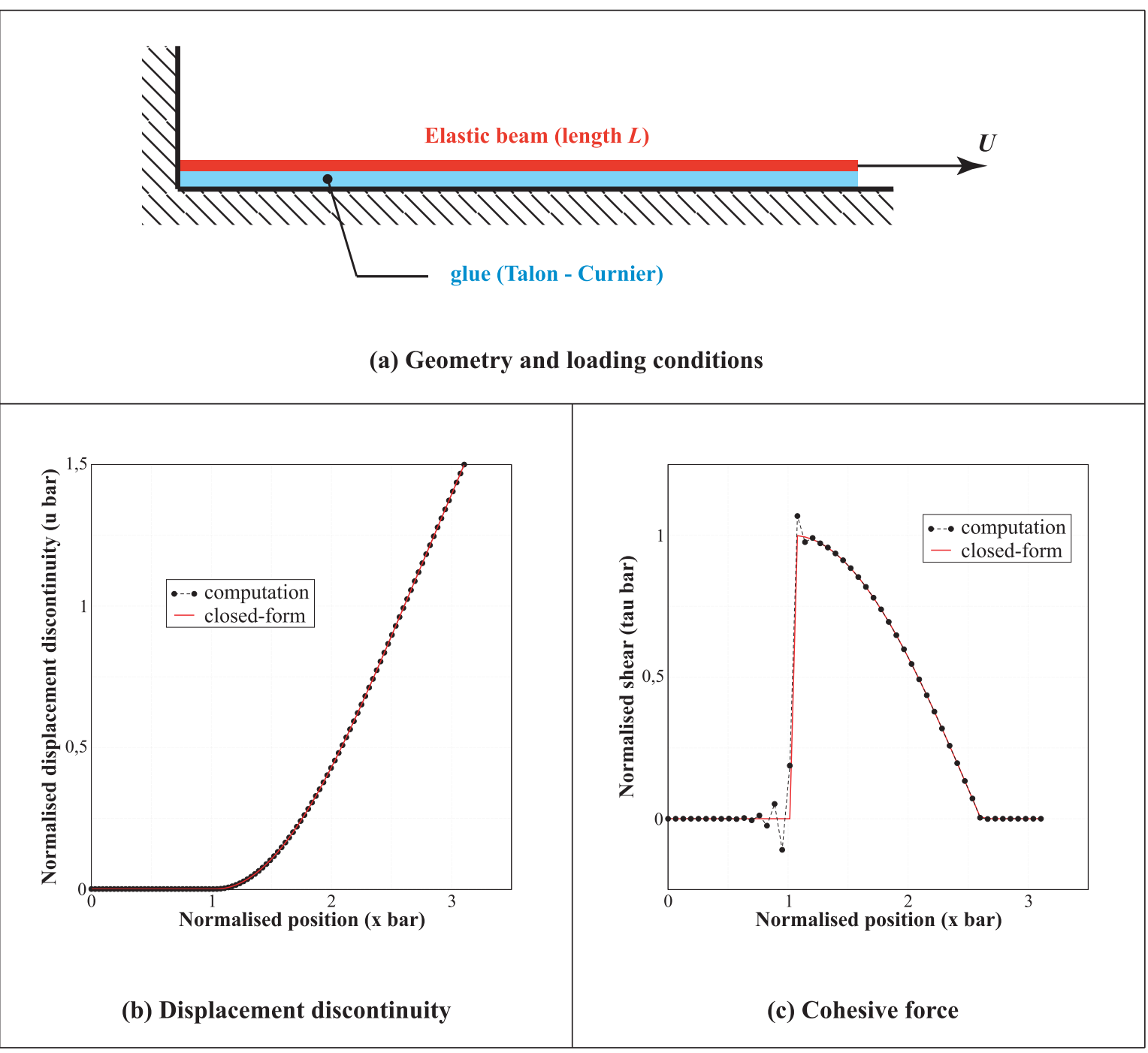

figure 4 - Validation test: an elastic beam glued on a rigid surface 


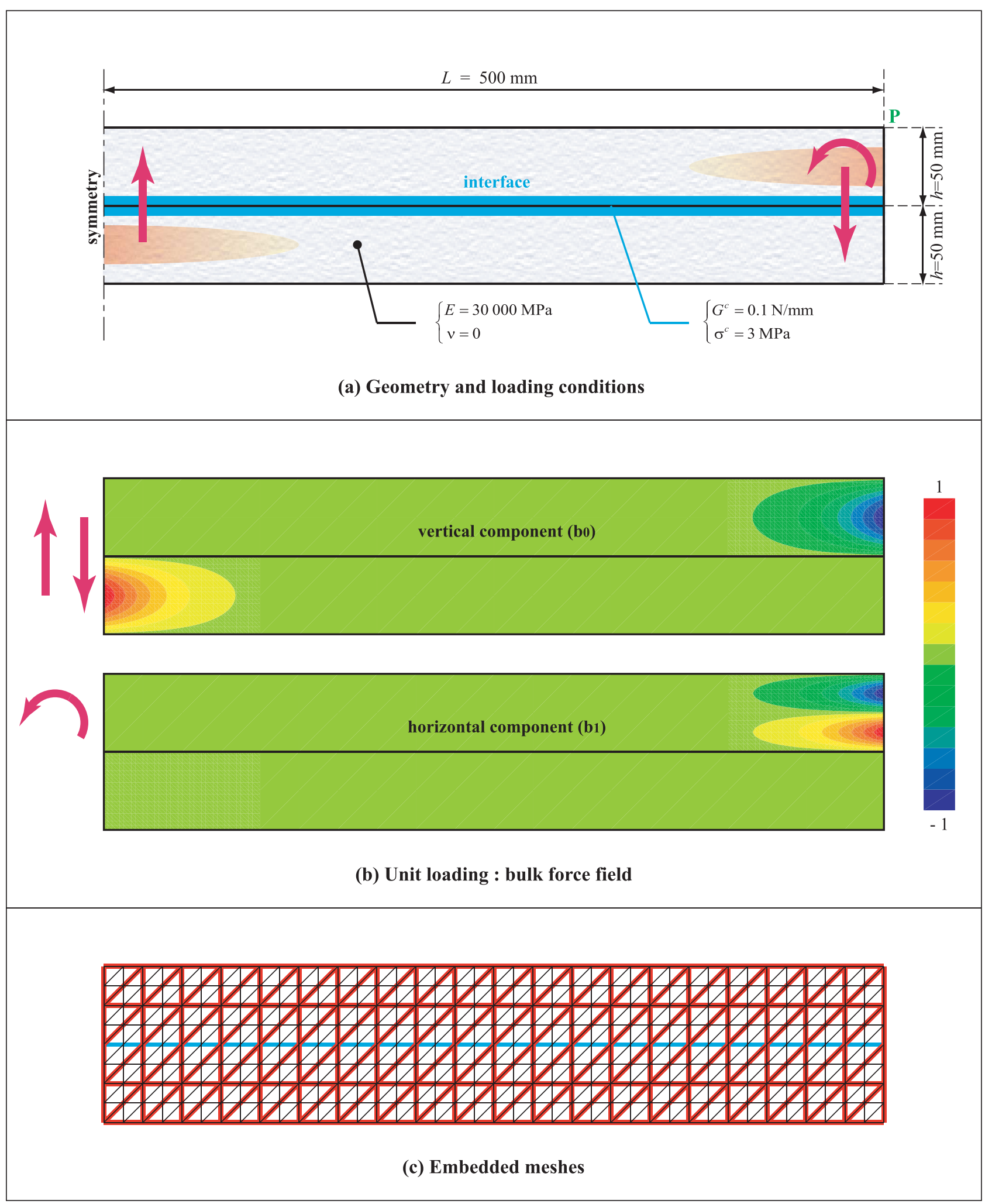

figure 5 - Description of the 2D sandwich beams problem 


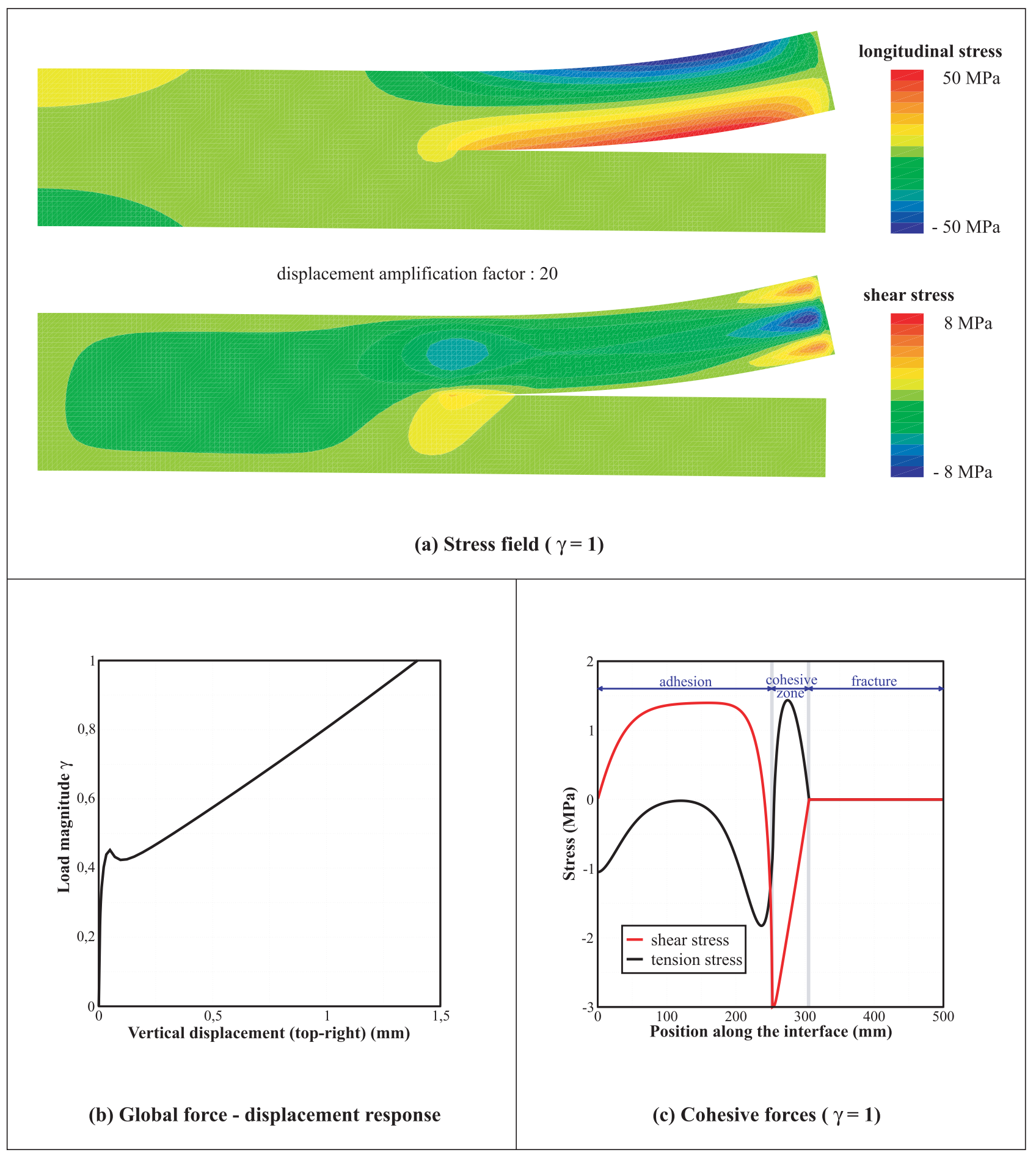




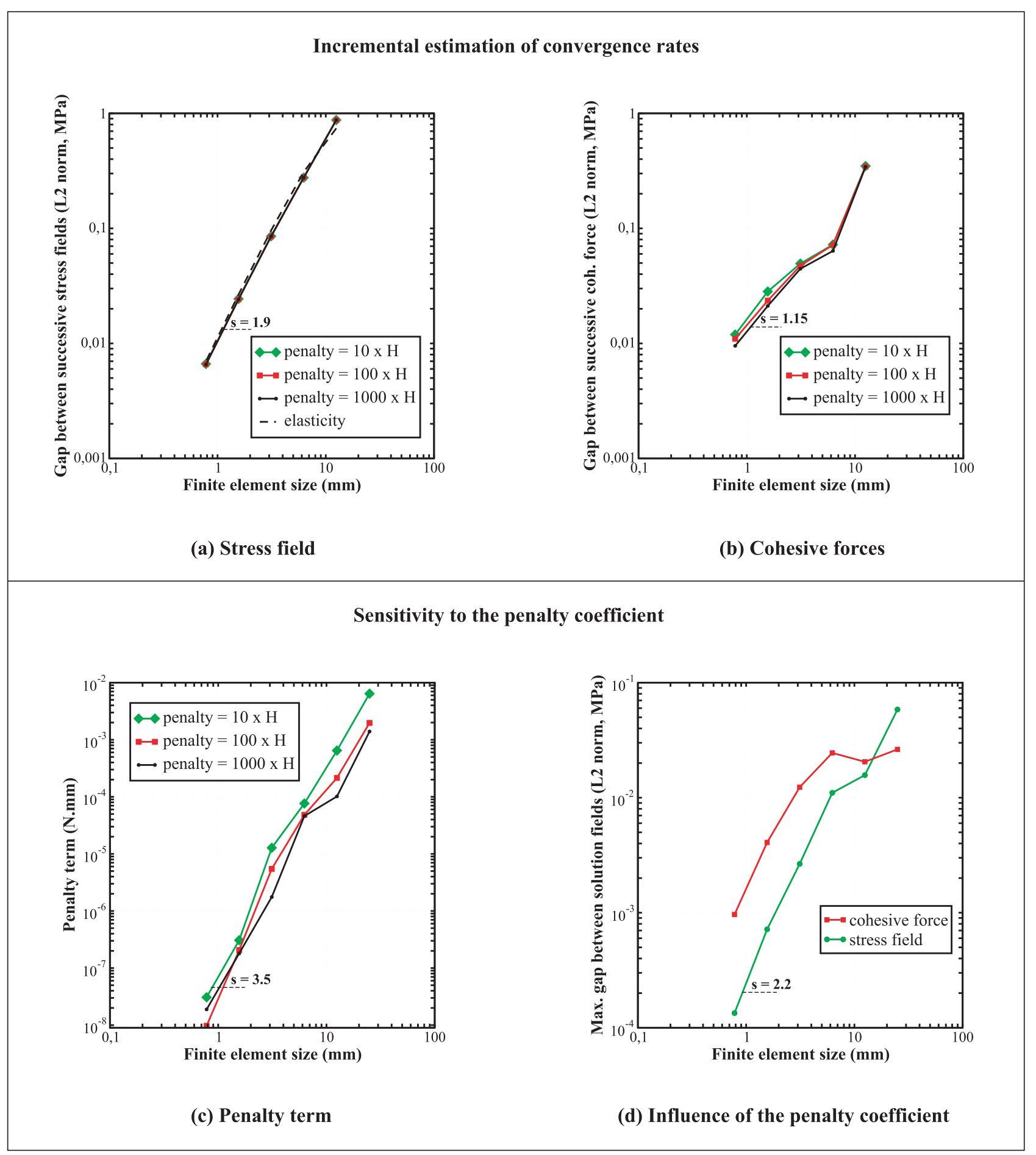




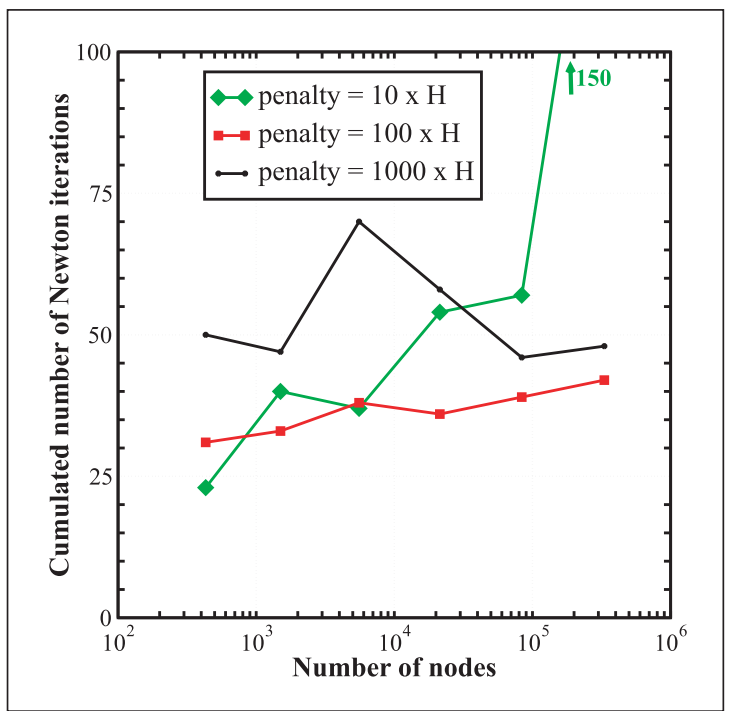

figure 8 - Convergence of Newton algorithm with respect to mesh size and penalty parameter 


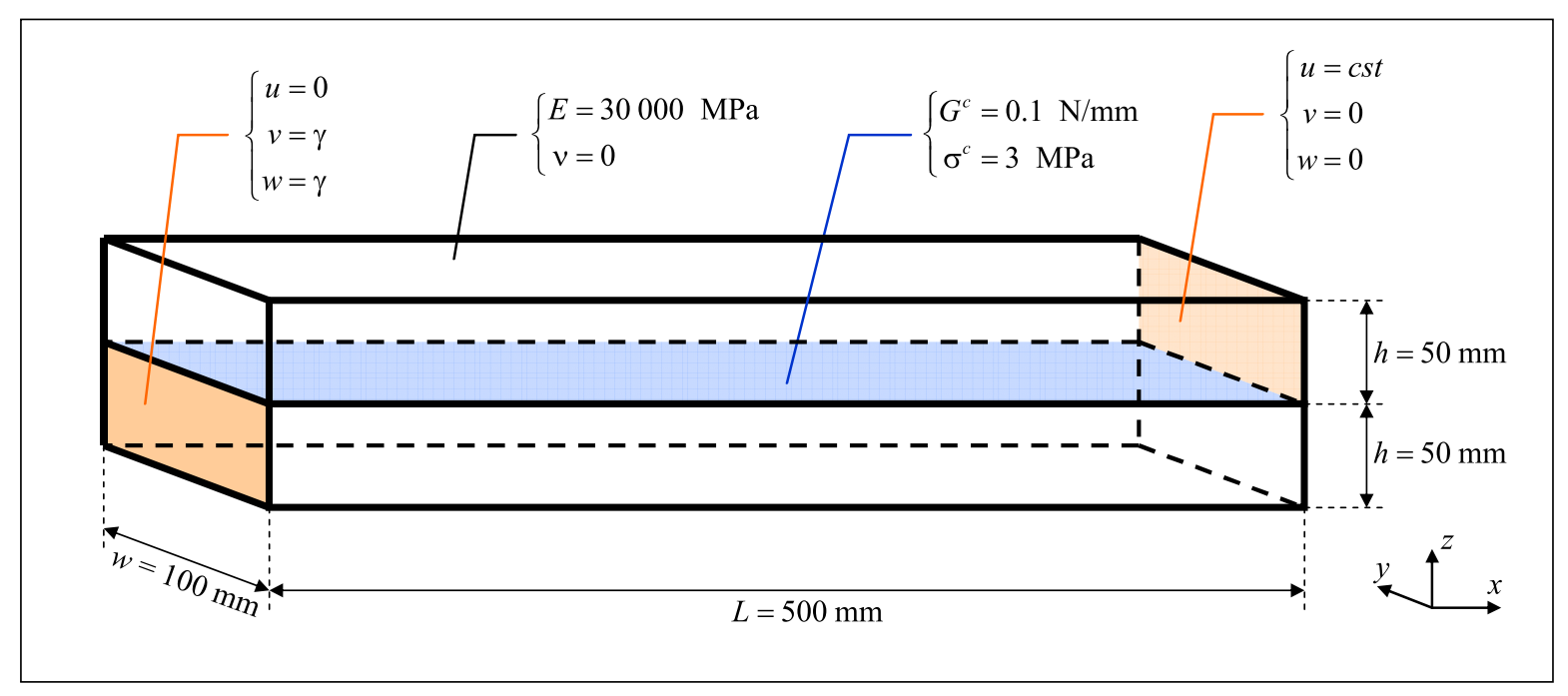

figure 9-Description of the 3D sandwich beams problem 


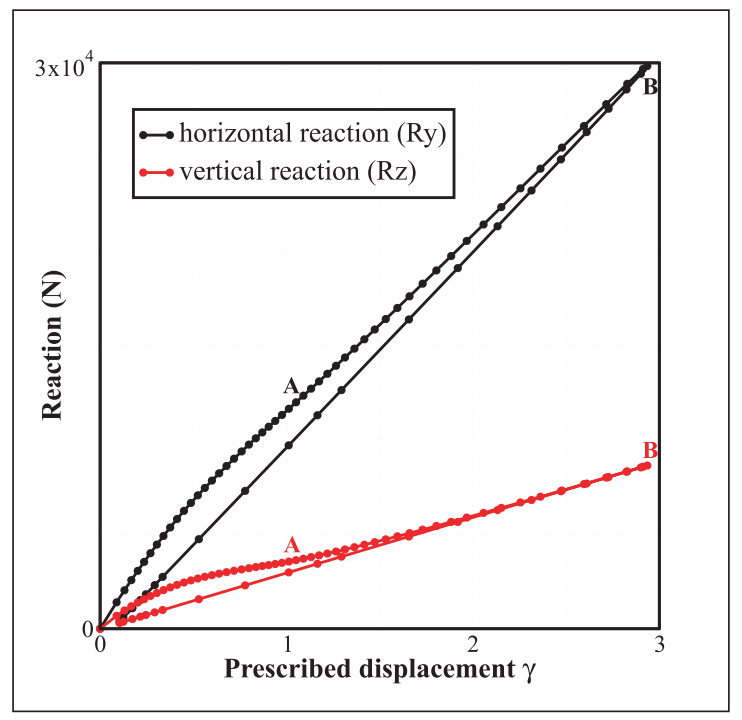

figure 10 - Global response of the 3D sandwich beams 


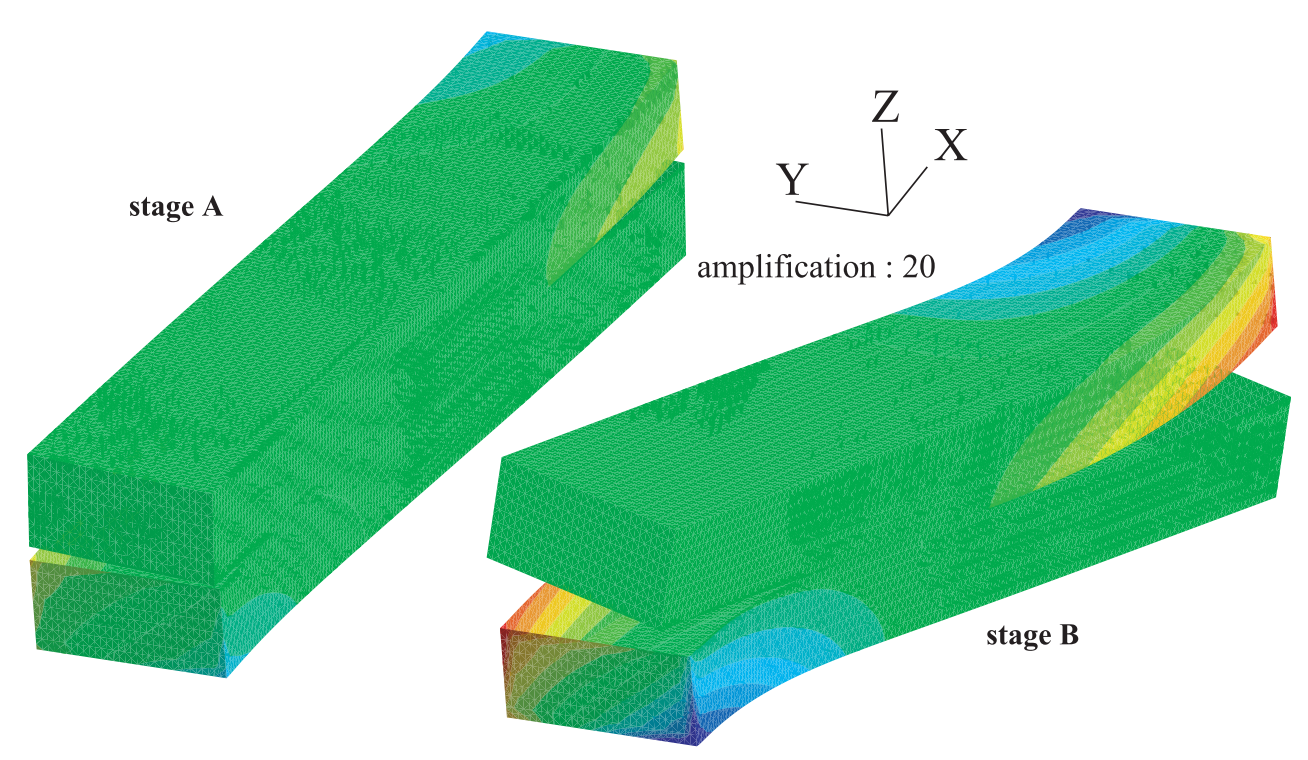

$150 \mathrm{MPa}$

(a) Deformed shape and longitudinal stress

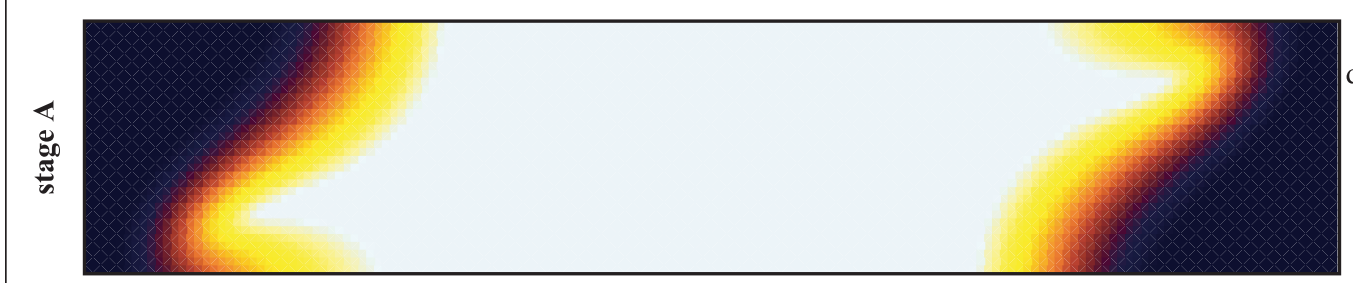

decohesion (1)

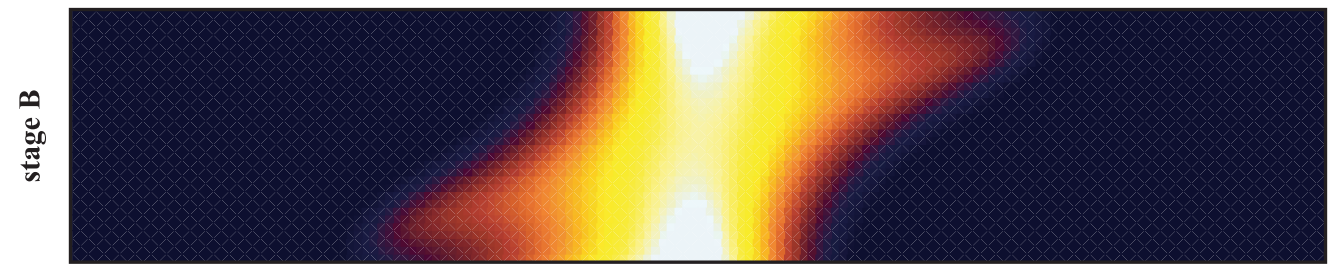

glued (0)

(b) Interfacial damage
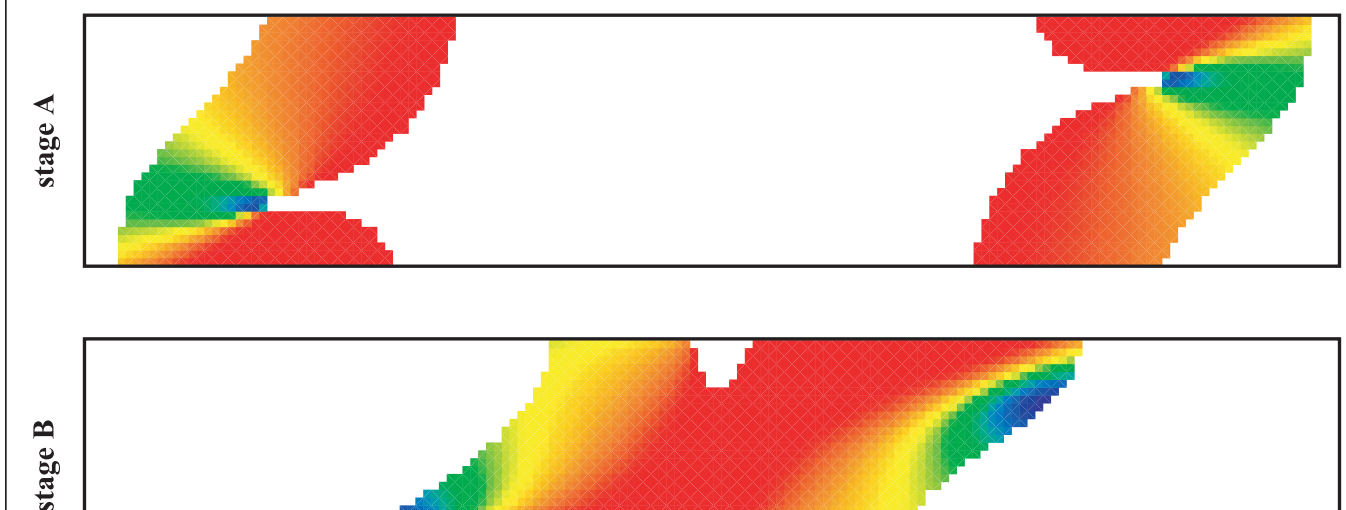

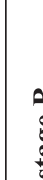

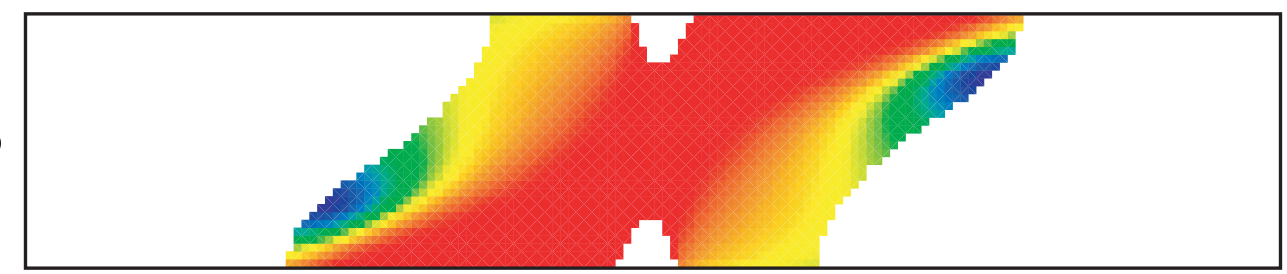

shear (1)

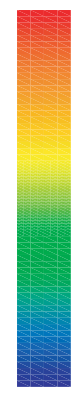

opening (0)

(c) Fracture mode inside the cohesive zone 


\section{table 1 - Spatial discretisation}

\begin{tabular}{|c|c|c|c|c|}
\hline Field & Shape functions & Unknowns & Interpolation & Finite element \\
\hline displacement & cont-P2 $[\mathbf{N}(\mathbf{x})]$ & nodal & $\mathbf{u}(\mathbf{x})=[\mathbf{N}(\mathbf{x})]\{\mathbf{U}\}$ & \\
\hline displ. discontinuity $\llbracket \mathbf{u} \rrbracket$ & cont-P2 $[\mathbf{D}(\mathbf{s})$ & nodal & $\llbracket \mathbf{u} \rrbracket(\mathbf{s})=[\mathbf{D}(\mathbf{s})]\{\mathbf{U}\}$ & \\
\hline Lagrange mult. & cont-P1 $[\mathbf{L}(\mathbf{s})]$ & nodal & $\lambda(\mathbf{s})=[\mathbf{L}(\mathbf{s})]\{\boldsymbol{\Lambda}\}$ & \\
\hline displ. discontinuity $\boldsymbol{\delta} \in \mathcal{D}_{h}$ & disc-P1 & Gauss points $\boldsymbol{\delta}_{g}$ & $\boldsymbol{\delta}\left(\mathbf{s}_{g}\right)=\boldsymbol{\delta}_{g}$ & middle plane \\
\hline
\end{tabular}

table 1 -Spatial discretisation 